\title{
INTERPRETING CLASSICAL THEORIES IN CONSTRUCTIVE ONES
}

\author{
JEREMY AVIGAD
}

\begin{abstract}
A number of classical theories are interpreted in analogous theories that are based on intuitionistic logic. The classical theories considered include subsystems of first- and second-order arithmetic. bounded arithmetic, and admissible set theory.
\end{abstract}

\$1. Introduction. Proof theory was developed, in part, as a way to reconcile classical and constructive aspects of mathematical reasoning. Given this historical fact, it is not surprising that over the years proof theorists have invested a good deal of effort in reducing classical theories to constructive ones.

Elegant in its simplicity, the Gödel-Gentzen double-negation interpretation works wonders. For example, it seamlessly reduces classical arithmetic to its intuitionistic counterpart, and with some additional work it can be used to interpret ZermeloFraenkel set theory in a variant based on intuitionistic logic [13]. Under the doublenegation interpretation, $\Pi_{2}$ sentences of arithmetic, which can be said to carry a theory's "computational" content, are not preserved, but in both these examples a further application of the Friedman-Dragalin interpretation [14] provides an easy and effective way of recovering the $\Pi_{2}$ theorems.

Unfortunately, the double-negation and Friedman-Dragalin translations do not always have desirable effects. For example, the double-negation translation of a classical axiom of choice is no longer an axiom of choice, and when applied to fragments of intuitionistic arithmetic, the Friedman-Dragalin translation increases the complexity of the formulae in the induction axioms. For more general purposes, ordinal analysis and functional interpretation are also powerful tools in the reductionist's kit. But though these approaches yield additional information, from the reductionist point of view the resulting interpretations are somewhat indirect.

In this paper I will discuss a way of "repairing" axioms that are damaged by the double-negation translation, using a method developed by Buchholz [7] and Coquand and Hofmann [11]. The history is as follows. In [7], Buchholz presented an interpretation of classical theories of iterated inductive definitions, reducing them to intuitionistic versions in which the inductive definitions are given by a strictly positive operator. These can then be further reduced to appropriate MartinLöf type theories, as in Palmgren [18], or to theories of "accessibility" inductive definitions, also in [7]. Recently, Coquand realized that Buchholz' treatment of

Received July 3, 1998; revised May 19, 1999.

Work partially supported by NSF Grant DMS 9614851. 
a single inductive definition can be adapted to yield a remarkably straightforward reduction of the fragment of arithmetic $I \Sigma_{l}$ to its intuitionistic version $I \Sigma_{l}^{i}$. In [11], Coquand and Hofmann also present an interpretation of Buss' theory of bounded arithmetic $S_{2}^{1}$, that is independent of (and somewhat different from) the one given here.

In Section 2, I will describe a general framework for these interpretations, and in the sections that follow this framework will be instantiated in a number of different settings. In Section 3, I will use it to give a slightly different presentation of Coquand's interpretation, which shows that $I \Sigma_{1}$ is conservative over its intuitionistic version for $\Pi_{2}$ formulae. I will then adapt the argument to $S_{2}^{l}$, yielding $\forall \Sigma_{1}^{b}$ conservation over its intuitionistic counterpart $I S_{2}^{l}$. Since $I \Sigma_{l}^{i}$ and $I S_{2}^{l}$ have constructive realizability interpretations, the reductions enable one to extract constructive information from the classical theories as well. The analysis also extends to full classical arithmetic, $P A$, and the theory $S_{2}$.

In Sections 4 and 5, I will consider the Kripke-Platek theory of admissible sets. Here the natural analogue of $I \Sigma_{l}$ is a variant of $K P$ in which foundation is restricted to formulae that are $\Sigma_{1}$ over the universe of sets. In Section 4, I will use an idea due to Friedman [13] to interpret this theory in an "intensional" version that omits the axiom of extensionality, and, in Section 5, I will apply the framework to interpret the latter in its intuitionistic counterpart.

We will ultimately obtain interpretations for versions of $K P$ with or without the axiom of infinity, and with either full or restricted foundation. The strongest theory analyzed in this way is $K P \omega$, which, by direct interpretation, encompasses a number of important classical theories. These include a theory of arithmetic inductive definitions, $I D_{I}$, and a subsystem of second-order arithmetic, $\Pi_{l}^{l}-C A^{-}$, which allows $\Pi_{1}^{1}$ comprehension without parameters (for details, see [8]). On the other hand, the intensional intuitionistic version IKP $\omega^{\text {int }}$ described below is contained in Aczel's constructive set theory, $C Z F$; the latter can, in turn, be embedded in an appropriate version of Martin-Löf type theory (see [2, 3]). In sum, the analysis below provides a net reduction of a number of classical theories to constructive ones.

In Section 6, I will show that the methods can also be applied in the context of subsystems of second-order arithmetic. In particular, we will see that the theory $\Sigma_{I}^{l}-A C$, which includes arithmetic comprehension and an axiom of choice for arithmetic formulae, is reducible to its intuitionistic counterpart, $\Sigma_{I}^{l}-A C^{i} . \Sigma_{I}^{I}-A C$ is interesting in its own right, but also interprets $\widehat{I D}_{I}$, which allows a weak form of arithmetic inductive definition. On the other hand, one can interpret $\Sigma_{l}^{I}-A C^{i}$ in an appropriate version of Martin-Löf type theory, ${ }^{1}$ so once again we have a net reduction of the classical theory to a constructive one.

I should emphasize that the proof-theoretic equivalences of the classical and intuitionistic theories discussed here are well-known. What is notable about this approach is that it applies uniformly to a wide range of theories, and does not require external "machinery." Because the approach involves effective translations

\footnotetext{
${ }^{1}$ More explicitly, Martin-Löf [17] shows that one can interpret $\Sigma_{I}^{I}-A C^{i}$ in a type theory with a single universe, without requiring an elimination rule on the universe. The interpretation is similar to that of the theory $I A R I$, described in [15, Section 6]. One obtains $\Sigma_{l}^{I}-A C^{i}$ from $I A R I$ by deleting the inductive generation axiom and substituting arithmetic choice for replacement; the choice schema is interpreted via a straightforward modification of Lemma 6.7 of [15].
} 
of the classical theories to the constructive ones, we obtain conservation results in which the increase in the lengths of proofs can be bounded by a polynomial; as far as I know, when it comes to $S_{2}^{l}$ and $I S_{2}^{l}$, this sharper form of the conservation result is new.

§2. The framework. One thing that makes intuitionistic logic attractive is that the logical connectives have a constructive interpretation, commonly attributed to Brouwer, Heyting, and Kolmogorov. What makes negation, in this context, particularly ornery is that it obliterates any constructive information that a formula might otherwise have held: the interpretation of $\theta \rightarrow \perp$ tells us nothing beyond the fact that there cannot possibly be a proof of $\theta$. The remedy offered by the FriedmanDragalin translation is to insist that $\perp$, along with the other atomic formulae, carry additional information; but the type of information these formulae carry is fixed in advance and remains static throughout the proof. The Buchholz-Coquand methods provide a more dynamic interpretation of $\perp$, by reinterpreting implication as well.

For our purposes it is convenient (but not necessary) to take intuitionistic logic to be given by a system of natural deduction, where derivations yield assertions of the form $\Gamma \Rightarrow \varphi$, i.e., " $\varphi$ follows from the hypotheses in $\Gamma$." To describe the method in full generality, let $L$ be any first-order language, and consider the following two-sorted "forcing language" $L_{f}$. One sort of $L_{f}$ has variables corresponding to the universe of $L$, with the associated constants and functions. The other sort has variables $p, q, r, \ldots$ ranging over "conditions," and there is a binary relation $p \preceq q$ (" $p$ is stronger than $q$ ") between objects this sort. Finally, for every $n$-ary relation symbol $R\left(x_{1}, \ldots, x_{n}\right)$ of $L$, there is a corresponding $(n+1)$-ary relation symbol $R^{\prime}\left(p, x_{1}, \ldots, x_{n}\right)$ of $L_{f}$. Intuitively, $R^{\prime}\left(p, x_{1}, \ldots, x_{n}\right)$ asserts that condition $p$ "forces" $R\left(x_{1}, \ldots, x_{n}\right)$. Then to every formula $\varphi$ in the language of $L$, we can inductively associate a formula $p \Vdash \varphi$ in the language of $L_{f}$, as follows:

$$
\begin{aligned}
p \Vdash R\left(t_{1}, \ldots, t_{n}\right) & \equiv R^{\prime}\left(p, t_{1}, \ldots, t_{n}\right), \quad \text { for every relation symbol } R \\
p \Vdash(\varphi \wedge \psi) & \equiv p \Vdash \varphi \wedge p \Vdash \psi \\
p \Vdash(\varphi \vee \psi) & \equiv p \Vdash \varphi \vee p \Vdash \psi \\
p \Vdash(\varphi \rightarrow \psi) & \equiv \forall q \preceq p(q \Vdash \varphi \rightarrow q \Vdash \psi) \\
p \Vdash \forall x \varphi & \equiv \forall x p \Vdash \varphi \\
p \Vdash \exists x \varphi & \equiv \exists x p \Vdash \varphi
\end{aligned}
$$

As usual, $\neg \varphi$ is defined to be $\varphi \rightarrow \perp$, and $\forall q \preceq p \theta$ is shorthand for $\forall q(q \preceq p \rightarrow \theta)$. $\perp$ is taken to be a 0 -ary relation, thereby covered by the first clause. If $\Gamma$ is a set of formulae $\left\{\psi_{1}, \ldots, \psi_{k}\right\}$, I will write $\Gamma, \varphi$ for $\Gamma \cup\{\varphi\}$, and $p \Vdash \Gamma$ for the set of formulae $\left\{p \Vdash \psi_{1}, \ldots, p \Vdash \psi_{k}\right\}$. The inscription $\Vdash \varphi$ is read " $\varphi$ is forced," and means that every condition forces $\varphi$.

Proposition 2.1 (substitution). For every formula $\varphi$, variable $x$, and term $t, p \Vdash$ $\varphi[t / x]$ is given by $(p \Vdash \varphi)[t / x]$.

DEFINITION 2.2. A forcing notion is good if it satisfies the following conditions:

1. $\preceq$ is reflexive and transitive.

2. The atomic forcing relations are monotone: $p \Vdash \theta$ and $p^{\prime} \preceq p$ implies $p^{\prime} \Vdash \theta$, for $\theta$ atomic. 
3. For every atomic formula $\theta$, if $p \Vdash \perp$ then $p \Vdash \theta$.

Notice that these conditions are expressible in $L_{f}$.

Proposition 2.3 (monotonicity). From the assumption that the forcing notion good, in intuitionistic logic one can prove monotonicity for all formulae in $L$.

Proof. By induction on formulae $\eta$. Monotonicity for atomic formulae takes care of the base case, and the transitivity of $\preceq$ is used when $\eta$ is of the form $\varphi \rightarrow \psi$.

Proposition 2.4. Let $\Gamma$ be a set of formulae in $L$, and $\varphi$ any formula in $L$. If $\varphi$ is provable from $\Gamma$ intuitionistically, then $p \Vdash \varphi$ is provable from $p \Vdash \Gamma$ and the assumption that the forcing notion is good.

Proof. Use induction on the length of the proof. We can assume that the only rule governing $\perp$ is "ex falso sequitur quodlibet" for atomic formulae; that is, the rule "From $\Gamma \Rightarrow \perp$ conclude $\Gamma \Rightarrow \theta$ " for atomic $\theta$. The case in which this is the last inference of the proof is covered by clause 3 in Definition 2.2.

Otherwise, the only interesting cases occur when the last inference is either an introduction or elimination rule for $\rightarrow$. To handle $\rightarrow$-introduction, suppose the last rule of the proof yields $\Gamma \Rightarrow \varphi \rightarrow \psi$ from $\Gamma, \varphi \Rightarrow \psi$. Assume $p \Vdash \Gamma, q \preceq p$, and $q \Vdash \varphi$; we need to show that $q \Vdash \psi$. By monotonicity, we have $q \Vdash \Gamma$; but then $q \Vdash \psi$ follows from the inductive hypothesis.

Dealing with $\rightarrow$-elimination is no more difficult.

Notes. The forcing clauses above formalize the usual Kripke semantics, provided $\perp$ is treated as a propositional variable and there is a fixed universe for all the possible worlds. See, for example, [23].

If $\preceq$ is defined from a commutative, idempotent "meet" operation $\wedge$ by

$$
q \preceq p \equiv(q \wedge p=q),
$$

then the clause for implication is equivalent to

$$
\forall q(q \Vdash \varphi \rightarrow p \wedge q \Vdash \psi) .
$$

If $\preceq$ has a greatest element $\emptyset$, then $\Vdash \varphi$ is equivalent to $\emptyset \Vdash \varphi$.

If one focuses one's attention on the negative fragment of intuitionistic logic, which involves only the connectives $\wedge, \rightarrow$, and $\forall$, the clauses above are consistent with the intuition that $p \Vdash \varphi$ means, in some sense, that there is a proof of $\varphi$ from $p$. We will see in Section 5 that the forcing relation behaves particularly well with respect to these connectives.

The Friedman-Dragalin translation arises from the clauses above in the special case where the partial order is trivial (i.e., has a single element) and there is a fixed formula $\psi$ such that for every atomic formula $\theta, p \Vdash \theta$ is just $\theta \vee \psi$.

To apply the framework above one need only find suitable interpretations for the conditions and atomic forcing relations.

COROLlary 2.5. Let T be an intuitionistic theory given by a set of axioms. Suppose one defines a good forcing notion in another theory $T^{\prime}$, in such a way that $T^{\prime}$ proves that every axiom of $T$ is forced. Then whenever $T$ proves a formula $\varphi, T^{\prime}$ proves that $\varphi$ is forced. 
Proof. If $T$ proves $\varphi$ intuitionistically, then there is a set of axioms $\Gamma$ of $T$ such that $\Gamma \Rightarrow \varphi$ is provable intuitionistically. If $p$ is any condition, the hypothesis and Proposition 2.4 imply that $T^{\prime}$ proves $p \Vdash \Gamma$ and hence $p \Vdash \varphi$.

$\S 3$. Arithmetic. In this section I will show that $I \Sigma_{l}$ is conservative over its intuitionistic analogue $I \Sigma_{l}^{i}$. The interpretation is essentially that of Coquand and Hofmann [11]; the only difference is that here I will use first-order forcing conditions instead of second-order ones, and divide the interpretation into two steps. In this form, it is easy to extend the results to $S_{2}^{l}$.

$I \Sigma_{l}$ denotes the fragment of classical arithmetic in which the schema of induction is restricted to $\Sigma_{1}$ formulae. Because any primitive recursive function can be introduced in a definitional extension of $I \Sigma_{l}$, we can conveniently blur the distinction between this theory and $I \Sigma_{l}(P R A)$, which has symbols denoting such functions in its language. Identifying primitive recursive relations with their characteristic functions, we can then take the $\Sigma_{1}$ formulae to be of the form $\exists x A(x)$, where $A$ is primitive recursive, possibly with free variables other than $x$. The axioms of $I \Sigma_{l}$ consist of

1. Quantifier-free defining equations for the primitive recursive functions.

2. $\Sigma_{1}$ induction: $\varphi(0) \wedge \forall x\left(\varphi(x) \rightarrow \varphi\left(x^{\prime}\right)\right) \rightarrow \forall x \varphi(x)$, where $\varphi$ is $\Sigma_{1}$ and $x^{\prime}$ denotes the successor of $x$.

$I \Sigma_{I}^{i}$ denotes the corresponding theory based on intuitionistic logic.

In $I \Sigma_{1}^{i}$ one can use induction to prove that equality is decidable, and hence that the law of the excluded middle holds for quantifier-free formulae. Markov's principle for primitive recursive predicates is given by

$$
\left(M P_{p r}\right) \quad \neg \forall x A(x) \rightarrow \exists x \neg A(x)
$$

where $A$ is primitive recursive. The interpretation of $I \Sigma_{l}$ in $I \Sigma_{l}^{i}$ will proceed in two steps: first we will interpret $I \Sigma_{l}$ in $I \Sigma_{l}^{i}+\left(M P_{p r}\right)$, and then we will interpret the latter theory in $I \Sigma_{l}^{i}$.

For the first interpretation, the double-negation interpretation suffices. In this context, the interpretation takes $(\varphi \vee \psi)^{N}$ to $\neg\left(\neg \varphi^{N} \wedge \neg \psi^{N}\right)$, takes $(\exists x \varphi)^{N}$ to $\neg \forall x \neg \varphi^{N}$, fixes atomic formulae, and commutes with $\wedge, \rightarrow$, and $\forall$.

LEMMA 3.1. I $\Sigma_{I}^{i}+\left(M P_{p r}\right)$ proves the double-negation translation of each axiom of $I \Sigma_{l}$.

Proof. Using $\left(M P_{p r}\right)$ and the law of the excluded middle for atomic formulae, $I \Sigma_{I}^{i}$ proves that quantifier-free and $\Sigma_{1}$ formulae are equivalent to their $\mathrm{N}$-translations. As a result, the doubly-negated axioms of $I \Sigma_{l}$ are equivalent to themselves in $I \Sigma_{l}^{i}+\left(M P_{p r}\right)$.

For the remainder of this paper I will say that a formula is "negative" if it is part of the negative fragment of intuitionistic logic, that is, it does not contain any instances of the connectives $\vee$ or $\exists .^{2}$ Let $\mathscr{C}_{p r}$ denote the set of "almost negative" formulae, that is, the smallest set containing the $\Sigma_{1}$ formulae and closed under conjunction, implication, and universal quantification. Since $I \Sigma_{I}^{i}+\left(M P_{p r}\right)$ proves that any formula in $\mathscr{C}_{p r}$ is equivalent to its $\mathrm{N}$-translation, we have

\footnotetext{
${ }^{2}$ The word "negative" is, unfortunately, overburdened in the literature; in particular, the usage here differs from that of [7].
} 
THEOREM 3.2. $I \Sigma_{l}$ is conservative over $I \Sigma_{l}^{i}+\left(M P_{p r}\right)$ for formulae in $\mathscr{C}_{p r}$.

Let us now apply the framework of Section 2 to interpret $I \Sigma_{I}^{i}+\left(M P_{p r}\right)$ in $I \Sigma_{l}^{i}$. It turns out that the appropriate conditions are finite sets

$$
\left\{\forall x A_{1}(x), \ldots, \forall x A_{k}(x)\right\}
$$

of $\Pi_{1}$ sentences with parameters, i.e., $\Pi_{1}$ formulae together with assignments to their free variables. ${ }^{3}$ The ordering $\preceq$ between conditions is defined to be the set containment relation, $\supseteq$. Fixing some reasonable encoding, if $\forall x A(x)$ is a $\Pi_{1}$ formula with free variables $\vec{y}$, let $\ulcorner\forall x A(x)\urcorner$ denote the function of $\vec{y}$ that returns the code of the corresponding sentence with parameters, and let the variables $p, q, r \ldots$ range over finite (coded) sets of such sentences. I will usually write $p, q$ instead of $p \cup q$ and $p, \varphi$ instead of $p \cup\{\varphi\}$. Since $q \supseteq p$ is equivalent to $q \cup p=q$, $p \Vdash(\varphi \rightarrow \psi)$ is equivalent to

$$
\forall q(q \Vdash \varphi \rightarrow p, q \Vdash \psi),
$$

which is the characterization I will use to verify the interpretations.

Given any proof in $I \Sigma_{l}^{i}+\left(M P_{p r}\right)$, choose $m$ large enough so that all the primitive recursive relations mentioned have complexity less than $m$, and let $\operatorname{Tr}_{p r}^{m}$ denote a primitive recursive truth predicate for such relations. (I will come back to this issue below.) If $p$ codes a set of the form (1), define

$$
\operatorname{Tr}(p, u) \equiv \bigwedge_{i=1}^{k} A_{i}(u),
$$

where $\operatorname{Tr}_{p r}^{m}$ is used to express the right-hand-side; this asserts that the formulae in $p$ are true at least as far as $u$ is concerned. Define

$$
p \vdash \varphi \equiv \exists u(\operatorname{Tr}(p, u) \rightarrow \varphi)
$$

for arbitrary formulae $\varphi$. Intuitively, the witness $u$ in (2) can be interpreted as a "proof" that $\varphi$ follows from the conjunction of the universal sentences in $p$, since (2) asserts that $\varphi$ follows more specifically from their instantiations at $u$. As one might expect, this "provability" relation is monotone in the first argument: if $q$ and $p$ are conditions such that $q \supseteq p$, then $\operatorname{Tr}(q, u)$ implies $\operatorname{Tr}(p, u)$ and hence $p \vdash \varphi$ implies $q \vdash \varphi$.

Define $p \Vdash \theta$ to be $p \vdash \theta$ when $\theta$ is atomic, and extend the forcing relation to arbitrary formulae in the language of arithmetic as in Section 2. It is not difficult to verify that the forcing notion is a good one, according to Definition 2.2. The following lemma shows that when it comes to quantifier-free formulae, the relations $\Vdash$ and $\vdash$ coincide.

Lemma 3.3. If $\varphi$ is any quantifier-free formula, then $I \Sigma_{l}^{i}$ proves

$$
p \Vdash \varphi \quad \text { iff } \quad p \vdash \varphi .
$$

\footnotetext{
${ }^{3}$ Alternatively one can take these to be $\Pi_{1}$ sentences in an expanded language that has a name for every element of the universe. Of course, in the context of arithmetic these names are not necessary, since every number is denoted by the corresponding numeral; but we will need this more general formulation in Sections 5 and 6 below.
} 
PROOF. The proof of this lemma relies heavily on the fact that $I \Sigma_{l}^{i}$ proves the law of the excluded middle for quantifier-free formulae. In particular, this implies that for such formulae $\varphi, p \vdash \varphi$ is equivalent to $\exists u(\neg \operatorname{Tr}(p, u) \vee \varphi)$, as well as $\exists u \neg \operatorname{Tr}(p, u) \vee \varphi$.

The proof proceeds by induction on the complexity of $\varphi$. The cases in which $\varphi$ is atomic, of the form $\theta \wedge \psi$, or of the form $\theta \vee \psi$ are readily dealt with, using the observations in the preceeding paragraph. When $\varphi$ is of the form $\theta \rightarrow \psi$ we have

$$
\begin{aligned}
p \Vdash(\theta \rightarrow \psi) & \leftrightarrow \forall q(q \Vdash \theta \rightarrow p, q \Vdash \psi) \\
& \leftrightarrow \forall q(q \vdash \theta \rightarrow p, q \vdash \psi) .
\end{aligned}
$$

We need to show that this is equivalent to $p \vdash(\theta \rightarrow \psi)$. In the forwards direction, assume the last line of the equivalence holds. Since $\theta$ implies $\emptyset \vdash \theta$, we have $\theta \rightarrow p \vdash \psi$. This is equivalent to

$$
\neg \theta \vee \exists u \neg \operatorname{Tr}(p, u) \vee \psi,
$$

which is equivalent to $p \vdash(\theta \rightarrow \psi)$. For the other direction, suppose $p \vdash(\theta \rightarrow \psi)$ and $q \vdash \theta$; we need to show $p, q \vdash \psi$. From the assumption, we have

$$
\exists u \neg \operatorname{Tr}(p, u) \vee(\theta \rightarrow \psi) \text { and } \exists v \neg \operatorname{Tr}(q, v) \vee \theta .
$$

Arguing by cases yields

$$
\exists w \neg \operatorname{Tr}(p \cup q, w) \vee \psi,
$$

as desired.

LEMMA 3.4. For every primitive recursive relation $A, I \Sigma_{l}^{i}$ proves the following:

1. $\forall x A(x) \Vdash \forall x A(x)$.

2. If $p \Vdash \neg \forall x A(x)$, then $p \Vdash \exists x \neg A(x)$.

Proof. For 1, we have by definition

$$
\begin{aligned}
\forall x A(x) \Vdash \forall x A(x) \text { iff } & \forall z(\forall x A(x) \Vdash A(z)) \\
\text { iff } & \forall z(\forall x A(x) \vdash A(z)) \\
\text { iff } & \forall z \exists u\left(\operatorname{Tr}_{p r}^{m}(\{\ulcorner\forall x A(x)\urcorner\}, u) \rightarrow A(z)\right) .
\end{aligned}
$$

Given $z$, simply take $u=z$.

For 2, suppose $p \Vdash \neg \forall x A(x)$. Then we have

$$
\forall q(q \Vdash \forall x A(x) \rightarrow p, q \Vdash \perp) .
$$

In particular, 1 implies $p, \forall x A(x) \Vdash \perp$. In other words, there is an element $u$ such that

$$
\operatorname{Tr}(p, u) \wedge A(u) \rightarrow \perp
$$

and hence

Taking $x=u$ we have

$$
\operatorname{Tr}(p, u) \rightarrow \neg A(u)
$$

which is, by definition,

$$
\exists x \exists u(\operatorname{Tr}(p, u) \rightarrow \neg A(x))
$$

$$
\exists x p \vdash \neg A(x) .
$$

By Lemma 3.3 this is equivalent to

$$
\exists x p \Vdash \neg A(x),
$$


which is, by definition, $p \Vdash \exists x \neg A(x)$.

LEMMA 3.5. I $\Sigma_{l}^{i}$ proves that each axiom of $I \Sigma_{l}^{i}+\left(M P_{p r}\right)$ is forced.

Proof. By Lemma 3.3, a quantifier-free formula is forced iff it is true, so the quantifier-free axioms are reduced to themselves. Lemma 3.4 shows that if $p$ forces the antecedent of $\left(M P_{p r}\right)$, then it forces the conclusion as well; so $\left(M P_{p r}\right)$ is forced.

Finally, to handle induction, suppose

$$
p \Vdash \psi(0) \quad \text { and } \quad p \Vdash \forall x\left(\psi(x) \rightarrow \psi\left(x^{\prime}\right)\right) .
$$

where $\psi(x)$ is $\Sigma_{1}$. The second assumption implies that for every $x$, if $p$ forces $\psi(x)$, then $p$ forces $\psi\left(x^{\prime}\right)$ as well. Since $p \Vdash \psi(x)$ is equivalent to a $\Sigma_{1}$ formula, we can use induction to show that for every $x, p$ forces $\psi(x)$; in other words $p \Vdash \forall x \psi(x)$. This shows that the induction axiom for $\psi$ is forced.

Let $\mathscr{D}_{p r}$ be the smallest set of formulae containing the quantifier-free ones and closed under conjunction, disjunction, and universal and existential quantification. For formulae $\varphi$ in $\mathscr{D}_{p r}, I \Sigma_{I}^{i}$ proves that $\Vdash \varphi$ is equivalent to $\varphi$. So we have

THEOREM 3.6. $I \Sigma_{l}^{i}+\left(M P_{p r}\right)$ is conservative over $I \Sigma_{l}^{i}$ for formulae in $\mathscr{D}_{p r}$.

This yields

THEOREM 3.7. $I \Sigma_{1}$ is conservative over $I \Sigma_{l}^{i}$ for $\Pi_{2}$ formulae.

PRoof. Every $\Pi_{2}$ formula is contained in $\mathscr{C}_{p r} \cap \mathscr{D}_{p r}$.

Since more general instances of induction translate to instances of induction, we have

THEOREM 3.8. PA and $H A+\left(M P_{p r}\right)$ are conservative over $H A$ for $\Pi_{2}$ formulae.

There is a correspondence between intermediate theories as well. Suppose we start with a $\Pi_{n}$ formula $\forall x_{1} \exists x_{2} \ldots Q x_{n} \varphi$, where $n \geq 2$ and $\varphi$ is quantifier-free. Then its double-negation translation is intuitionistically equivalent to $\forall x_{1} \neg \exists x_{2} \ldots Q^{\prime} x_{n} \neg \varphi^{N}$, where $Q^{\prime}$ is $\exists$ if $Q$ is $\forall$, and vice-versa. By definition, the assertion that $p$ forces this latter formula is equivalent to

$$
\forall x_{1}, q\left(q \Vdash \exists x_{2} \ldots Q^{\prime} x_{n} \neg \varphi^{N} \rightarrow p \cup q \Vdash \perp\right),
$$

and this formula is equivalent (over, say $\left.I \Sigma_{1}^{i}\right)$ to one of the form $\forall\left(\Sigma_{n} \rightarrow \Sigma_{1}\right)$. For each $n \geq 2, C_{n}$ be the set of formulas of this form. Since from a classical point of view formulas in $C_{n}$ are $\Pi_{n}$, and since $\Pi_{n}$ and $\Sigma_{n}$ induction are equivalent in the classical setting, we have

THEOREM 3.9. For $n \geq 2, I \Sigma_{n}$ is a $\Pi_{2}$ conservative extension of $I C_{n}^{i}$.

More satisfying results involving the correspondence between classical and intuitionistic fragments of arithmetic are available; see Burr [9].

The argument we have just carried out required a primitive recursive truth predicate $\operatorname{Tr}_{p r}^{m}$ for primitive recursive relations of complexity less than or equal to $m$. If we measure the complexity of a primitive recursive function by the number of instances of composition and primitive recursion employed in its definition, we can obtain such a predicate as follows. For each $m$, we define a primitive recursive function $\operatorname{Eval}^{m}(f, s)$, which evaluates a function of complexity at most $m$, coded by $f$, at the list of parameters coded by $s$. Eval ${ }^{0}$ is defined so that it computes the result of applying either a constant, successor, or projection function to its arguments, and then for each $m, \mathrm{Eval}^{m+1}$ is defined by cases using $\mathrm{Eval}^{m}$. (A similar construction 
can be found in [22, Section 1.5].) We can use Eval ${ }^{m}$ to define $\operatorname{Tr}_{p r}^{m}$, and derive the necessary properties in $I \Sigma_{l}^{i}$.

Alternatively, we can use a $\Sigma_{1}$ evaluation predicate for the primitive recursive functions (also described in [22]) and take $p \vdash \theta$ to assert that "there is a $u$ and a computation sequence $c$ for $A_{1}(u), \ldots, A_{k}(u)$, such that if the computation determines that these are all true, then $\theta$." Choosing this method means that we no longer need to use a separate interpretation for each fixed complexity level $\mathrm{m}$.

For the simplest method of all, note that in any given proof only finitely many relations $A_{1}, \ldots, A_{k}$ are mentioned, in which case the "truth predicate" need only work for $\Pi_{1}$ sentences involving these relations. We can represent every such sentence as a pair $\langle i, s\rangle$, where $i$ is a value between 1 and $k$ and $s$ codes an assignment to the free variables of $\forall x A_{i}(x)$. With this representation, $\operatorname{Tr}_{p r}^{m}$ can be defined as a straightforward disjunction of length $k$, and to carry out the interpretation we only need a minimal theory of finite sets and sequences in $I \Sigma_{l}^{i}$.

In the applications that follow, one may be able to use variations of each of these three options. Since the last one requires the least effort and is the most clearly applicable in all cases, let us, for concreteness, adopt this way of interpreting the various forms of $\operatorname{Tr}^{m}$ referred to in the sequel. Though I will neglect to qualify the statements of the lemmata below with the condition that certain formulae mentioned must have complexity less than $m$, the reader can readily supply the additional details.

With these considerations in hand the interpretation above can be adapted to Buss' theory of bounded arithmetic $S_{2}^{l}$, and its constructive counterpart, $I S_{2}^{l}$. I will rely on the presentation in Cook and Urquhart [10], which provides a nice account of these two theories and their properties. In fact, it will be more convenient to work with the theories $C P V$ and $I P V$, which are definitional extensions of $S_{2}^{l}$ and $I S_{2}^{l}$ respectively; these extensions include the terms of Cook's theory $P V$, which denote polynomial time computable functions. Bounded quantification is defined by

$$
\forall x \leq t \varphi \equiv \forall x\left(t^{\prime} \leq x \vee \varphi\right)
$$

and

$$
\exists x \leq t \varphi \equiv \exists x(x \leq t \wedge \varphi) .
$$

In analogy to the $\Sigma_{1}$ formulae above, a formula is said to be $N P$ if it is of the form $\exists x \leq t A(x)$, where $A$ is a $P V$ relation and $t$ is a $P V$ term.

$C P V$ is the classical first-order theory given by

1. Quantifier-free axioms defining the basic symbols of the language.

2. $N P$ induction:

$$
\varphi(0) \wedge \forall x(\varphi(\lfloor x / 2\rfloor) \rightarrow \varphi(x)) \rightarrow \forall x \varphi(x)
$$

where $\varphi$ is $N P$.

$I P V$ is simply $C P V$ based on intuitionistic logic. From [10, Theorem 4.5] we know that $I P V$ proves the law of the excluded middle for quantifier-free formulae (and, in fact, formulae in which every quantifier is sharply bounded). In particular, we can take the double-negation translation to fix atomic formulae, and show that the translation of a bounded formula is equivalent to a bounded formula. 
A bounded analogue of Markov's principle is given by $\left(M P_{b}\right)$

$$
\neg \forall x \leq t A(x) \rightarrow \exists x \leq t \neg A(x)
$$

where $A$ is a $P V$ relation.

Finally, in analogy to the almost negative formulae, define $\mathscr{C}_{b}$ to be the smallest set of formulae containing the $N P$ formulae and closed under conjunction, implication, and universal quantification. The double-negation interpretation yields

THEOREM 3.10. $C P V$ is a conservative extension of $I P V+\left(M P_{b}\right)$ for formulae in $\mathscr{C}_{b}$.

The interpretation of $I P V+\left(M P_{b}\right)$ in $I P V$ follows the pattern of the interpretation of $I \Sigma_{l}^{i}+\left(M P_{p r}\right)$ in $I \Sigma_{l}^{i}$, once we take conditions to be finite sets of sentences of the form $\forall x \leq b A(x)$, where $A(x)$ is a relation in $P V$ that may have parameters, and $b$ is a parameter. Let $\operatorname{Tr}_{b}^{m}$ be a truth predicate for a sufficiently large set of $P V$ relations with parameters. If $p$ is the set

$$
\left\{\forall x \leq b_{1} A_{1}(x), \ldots, \forall x \leq b_{k} A_{k}(x)\right\},
$$

use $\operatorname{Tr}_{b}^{m}$ and to define

$$
\operatorname{Tr}(p, u) \equiv \bigwedge_{i=1}^{k}\left(u \leq b_{i} \rightarrow A_{i}(u)\right)
$$

and

$$
p \vdash \varphi \equiv \exists u \leq \max \left(b_{1}, \ldots, b_{k}\right)(\operatorname{Tr}(p, u) \rightarrow \varphi)
$$

for arbitrary $\varphi$. Finally, define $p \Vdash \theta$ to be $p \vdash \theta$ for atomic $\theta$, and then extend the forcing relation to the entire language of $I P V$.

The following lemma asserts that bounded quantification commutes with the $\Vdash$ operator.

LEMMA 3.11. For every formula $\varphi, I P V$ proves the following:

1. $p \Vdash \exists x \leq t \varphi$ iff $\exists x \leq t p \Vdash \varphi$.

2. $p \Vdash \forall x \leq t \varphi$ iff $\forall x \leq t p \Vdash \varphi$.

Proof. As in the proof of Lemma 3.3, if $\theta$ is atomic then $p \Vdash \theta$ is equivalent to $\theta \vee p \Vdash \perp$. As a result, we have

$$
\begin{array}{ll}
p \Vdash \exists x \leq t \varphi & \text { iff } \quad \exists x(p \Vdash x \leq t \wedge p \Vdash \varphi) \\
& \text { iff } \quad \exists x((x \leq t \vee p \Vdash \perp) \wedge p \Vdash \varphi) .
\end{array}
$$

We need to show that this is equivalent to $\exists x \leq t p \Vdash \varphi$. One direction is easy; for the other direction, suppose the last line of the equivalence above holds. Given such a value of $x$, reason by cases: if $x \leq t$, we are done. If, on the other hand, $p \Vdash \perp$, then $p \Vdash \varphi[0 / x]$, since intuitionistic validities are forced; and again we are done.

The argument for 2 is similar.

In analogy with Lemmata 3.3-3.5 we have

LEMMA 3.12. If $\varphi$ is any quantifier-free formula, then IPV proves

$$
p \Vdash \varphi \text { iff } p \vdash \varphi \text {. }
$$

Lemma 3.13. For any $P V$ predicate $A, I P V$ proves the following:

1. $\forall x \leq t A(x) \Vdash \forall x \leq t A(x)$.

2. If $p \Vdash \neg \forall x \leq t A(x)$, then $p \Vdash \exists x \leq t \neg A(x)$. 
LEMMA 3.14. IPV proves that each axiom of $I P V+\left(M P_{b}\right)$ is forced.

Let $\mathscr{D}_{b}$ be the smallest set of formulae in the language of IPV containing the quantifier-free ones and closed under conjunction, disjunction, and universal and existential quantification.

THEOREM 3.15. IPV $+\left(M P_{b}\right)$ is conservative over IPV for formulae in $\mathscr{D}_{b}$.

THEOREM 3.16. CPV is conservative over IPV for $\forall N P$ formulae, that is, formulae of the form $\forall x \exists y \leq t A(x, y)$, where $A$ is a $P V$ relation.

Using the analysis in [10], which shows that $C P V$ and $I P V$ are definitional extensions of $S_{2}^{l}$ and $I S_{2}^{l}$, respectively, this yields

THEOREM 3.17. $S_{2}^{l}$ is conservative over I $S_{2}^{l}$ for $\forall \Sigma_{1}^{b}$ formulae.

Let $S_{2}$ and $I S_{2}$ denote the extensions of $S_{2}^{1}$ and $I S_{2}^{1}$ in which induction is allowed for arbitrary bounded formulae. Using Lemma 3.11, we see that Lemma 3.14 still holds for this strengthened form of induction, so we have

THEOREM 3.18. $S_{2}$ is conservative over $I S_{2}$ for $\forall \Sigma_{1}^{b}$ formulae.

In [11], Coquand and Hofmann interpret $C P V$ in a second-order version of $I P V$, and then invoke a result from [10] which reduces this to first-order $I P V$. Their methods yield a strengthening of Theorem 3.17, where the class of formulas conserved include those of the form $\forall \exists \Sigma_{1}^{b}$. On the other hand, because the conservation result from [10] uses a normalization argument, there is the possibility of a superexponential increase in the lengths of proofs. Alternatively, one can derive the stronger conclusion from Theorem 3.17 using Parikh's theorem [19], but once again this allows for superexponential growth. As far as I know, it is still an open question as to whether one can obtain the strengthened version of Theorem 3.17 with a polynomial bound on the increase in the lengths of proofs.

\$4. Extensionality in admissible set theory. In the context of set theory, the proper analogue of $I \Sigma_{l}$ is the Kripke-Platek theory of admissible sets, without the axiom of infinity, and with foundation restricted to $\Sigma_{1}$ formulae: in addition to the fact that the two theories can be interpreted in one another, one finds additional structural similarities in the work of Rathjen [20].

I will take the language of set theory to contain only a single binary relation symbol $\in$, and take equality to be defined by

$$
x=y \equiv \forall z \in x(z \in y) \wedge \forall z \in y(z \in x) .
$$

Here bounded quantification is given by

$$
\forall y \in x \varphi \equiv \forall y(y \in x \rightarrow \varphi)
$$

and

$$
\exists y \in x \varphi \equiv \exists y(y \in x \wedge \varphi) .
$$

A formula is said to be $\Delta_{0}$, or restricted, if every quantifier is bounded; it is said to be $\Sigma_{1}\left(\right.$ resp. $\left.\Pi_{1}\right)$ if it is either $\Delta_{0}$ or of the form $\exists y \varphi($ resp. $\forall y \varphi)$, where $\varphi$ is $\Delta_{0}$. The axioms of $K P$ are as follows:

1. Extensionality: $x=y \rightarrow(x \in w \rightarrow y \in w)$.

2. Pair: $\exists x(y \in x \wedge z \in x)$.

3. Union: $\exists x \forall z \in y \forall w \in z(w \in x)$. 
4. $\Delta_{0}$ separation: $\exists x(\forall z \in x(z \in y \wedge \varphi(z)) \wedge \forall z \in y(\varphi(z) \rightarrow z \in x))$, where $\varphi$ is $\Delta_{0}$ and $x$ does not occur in $\varphi$.

5. $\Delta_{0}$ collection: $\forall x \in z \exists y \varphi(x, y) \rightarrow \exists w \forall x \in z \exists y \in w \varphi(x, y)$, where $\varphi$ is $\Delta_{0}$.

6. Foundation: $\forall x(\forall y \in x \psi(y) \rightarrow \psi(x)) \rightarrow \forall x \psi(x)$, for arbitrary $\psi$.

I have written $\Delta_{0}$ separation to emphasize that it is $\Sigma_{1}$; this axiom can be used to derive the more usual forms of pair and union. The foundation axiom as presented here is sometimes also called "set induction," and is equivalent to the assertion that every nonempty definable class of sets has an $\in$-least element. I will use $K P \uparrow$ to denote the theory in which foundation is restricted to $\Sigma_{1}$ formulae.

The definition of equality given above corresponds to the usual notion of extensional equality between sets. It is easy to prove that this relation is reflexive, symmetric, and transitive; and from axiom 1 we can derive $x=y \rightarrow(\varphi(x) \leftrightarrow \varphi(y))$ for every formula $\varphi$. Alternatively, we could have taken equality to be a basic logical symbol having these properties, and then replaced axiom 1 with what was previously the definition. The two approaches are equivalent, and the first is more convenient for our purposes.

We would like to interpret $K P$ and $K P\lceil$ in intuitionistic versions. One problem that we will encounter is that extensionality is not well-behaved under the doublenegation translation. So let us take $K P^{\text {int }}$ and $K P^{\text {int }} \uparrow$ to be "intensional" versions, in which the axiom of extensionality is omitted, and let us consider what life in an intensional universe might be like. One can think of such a universe as consisting of "names" for sets, where, in particular, there may be many names for the empty set; i.e., there may be two sets $x$ and $y$ satisfying $\forall z(z \notin x)$ and $\forall z(z \notin y)$, while for some $w$ we have $x \in w$ but $y \notin w$. Also, taking $x=\{y, z\}$ to abbreviate

$$
y \in x \wedge z \in x \wedge \forall w \in x(w=y \vee w=z)
$$

is misleading, since it is consistent that $z=\{x, y\}$ and $z^{\prime}=\{x, y\}$ while $z \neq z^{\prime}: z$ and $z^{\prime}$ may contain different names for $x$ and $y$.

Friedman [13] (see also Chapter VIII of [6]) has found an elegant way of interpreting extensionality in an intensional universe: declare all the empty sets to be "isomorphic" to each other, and, more generally, call two sets isomorphic if (inductively) they have isomorphic elements; then replace elementhood by elementhood up to isomorphism. Here I will show that this approach can be implemented in $K P^{\text {int }} \uparrow$.

A formula is said to be $\Delta_{1}$ (relative to a theory) if it is provably equivalent to both $\Sigma_{1}$ and $\Pi_{1}$ formulae. The next lemma is standard in admissible set theory, and does not require extensionality.

LEMMA 4.1. KPint $\uparrow$ proves collection for $\Sigma_{1}$ formulae, and separation for $\Delta_{1}$ formulae. Also, if $\varphi(R)$ is $\Delta_{0}$ in a language with a new relation symbol $R$, and one replaces $R$ with a $\Delta_{1}$ formula, the result is $\Delta_{1}$ in $K P^{\text {int }} \uparrow$.

Proor. One obtains $\Sigma_{1}$ collection by pairing the existentially quantified variables. For $\Delta_{1}$ separation, note that if $\varphi(y)$ is equivalent to a $\Sigma_{1}$ formula $\exists u \psi(y, u)$ as well as to a $\Pi_{1}$ formula $\forall u \theta(y, u)$, then (classically) we have $\forall y \exists u(\psi(y, u) \vee \neg \theta(y, u))$; one can then reduce separation for $\varphi$ to an instance of $\Delta_{0}$ separation by first using collection to gather a sufficiently large set of witnesses. The last claim is proved by induction on formulae, again using collection. For details, see [5]. 
Ignoring the caveat above and using $x=\{y, z\}$ to denote (3), I will write " $x$ is an unordered pair" for $\exists y \in x, z \in x(x=\{y, z\})$, and $\{y, z\} \in w$ for $\exists x \in w(x=$ $\{y, z\})$. We can think of a symmetric relation $R$ as given by a set of unordered pairs, allowing $\{x, x\}$ as a degenerate case. With this in mind, let us write $y \sim_{R} z$ for $\{y, z\} \in R$, and $y \in$ field $(R)$ for $\exists x \in R \exists z \in x(x=\{y, z\})$. Call such a relation $R$ an isomorphism relation if, for every $y$ and $z$ in the field of $R$, we have

$$
y \sim_{R} z \leftrightarrow\left(\forall u \in y \exists v \in z\left(u \sim_{R} v\right) \wedge \forall v \in z \exists u \in y\left(v \sim_{R} u\right)\right) .
$$

The definition implies that the field of any isomorphism relation $R$ is transitively closed, i.e., $y \in$ field $(R)$ and $w \in y$ imply $w \in$ field $(R)$. Using foundation with $\Delta_{0}$ formulae one can also show that any isomorphism relation is an equivalence relation on its field.

The global isomorphism relation we are looking for is given by

$$
y \sim z \equiv \exists R \text { (" } R \text { is an isomorphism relation and } y \sim_{R} z \text { "). }
$$

Each of the following four lemmata is provable in $K P^{i n t} \uparrow$. The last shows that $y \sim z$ has an equivalent $\Pi_{1}$ definition, and is hence $\Delta_{1}$.

LEMMA 4.2. KP int $\uparrow$ proves that for every $u$ and $v$, there is a set of unordered pairs of elements from $u$ and $v$; that is, for every $u$ and $v$,

$$
\exists w \forall y \in u, z \in v(\{y, z\} \in w) .
$$

Proof. Fix $u$ and $v$. For any given $y$, we have

$$
\forall z \in v \exists r(r=\{y, z\}) .
$$

Use $\Delta_{0}$ collection to obtain

$$
\exists s \forall z \in v(\{y, z\} \in s) .
$$

In particular, this is true for every $y$ in $u$; use $\Delta_{0}$ collection again to show

$$
\exists t \forall y \in u \exists s \in t \forall z \in v(\{y, z\} \in s) .
$$

Apply the union axiom to $t$ to get the desired $w$.

Say that an isomorphism relation is good for a set $x$ if every element of $x$ is in the field of $R$.

LEMMA 4.3. For every set $x$, there is an isomorphism relation that is good for $x$.

Proof. Use $\Sigma_{1}$ induction on $x$. Suppose the claim is true for every element of $x$; in other words, for every $y$ in $x$ there is an isomorphism relation $R_{y}$ good for $y$. Using collection and union, we can define $\hat{R}$ to be the union of the $R_{y}$. Using Lemma 4.2 and $\Delta_{0}$ separation, let $S$ contain unordered pairs from $x$ satisfying the right side of (4), with $\hat{R}$ in place of $R$; and let $R$ be $\hat{R} \cup S$. Then $R$ is an isomorphism relation that is good for $x$.

LEMMA 4.4. Suppose $R$ and $R^{\prime}$ are isomorphism relations. Then for every $y$ and $z$ in both the fields of $R$ and $R^{\prime}, y \sim_{R} z$ if and only if $y \sim_{R^{\prime}} z$.

Proof. Fix $R$ and $R^{\prime}$. Since the second sentence of the lemma is equivalent to a $\Delta_{0}$ assertion in $y$ and $z$, the result follows from a double induction on $y$ and $z$, and the definition of an isomorphism relation. 
LEMMA 4.5. $y \sim z$ is equivalent to the assertion $\forall R\left((\right.$ " $R$ an isomorphism relation" $\wedge y \in$ field $(R) \wedge z \in f$ field $\left.(R)) \rightarrow y \sim_{R} z\right)$.

Proof. Fix $y$ and $z$. The pairing axiom and Lemma 4.3 imply that there is an isomorphism relation with $y$ and $z$ in its field, and Lemma 4.4 implies that any two such relations must agree.

We have established that $\sim$ is a $\Delta_{1}$ relation. Using the definition and lemmata above one can show that $\sim$ satisfies

$$
y \sim z \leftrightarrow(\forall u \in y \exists v \in z(u \sim v) \wedge \forall v \in z \exists u \in y(v \sim u)) .
$$

Now define

$$
y \in^{*} w \equiv \exists z \in w(y \sim z),
$$

and if $\varphi$ is any formula in the language of set theory, let $\varphi^{*}$ denote the formula obtained by replacing $\in$ by $\in^{*}$. Observe that $(x=y)^{*}$ is given by

$$
\forall z \in^{*} x\left(z \in^{*} y\right) \wedge \forall z \in^{*} y\left(z \in^{*} x\right) .
$$

LeMma 4.6. Let $\varphi$ be any formula. The following are provable in KP $P^{\text {int }} \uparrow$ :

1. $x \in z \rightarrow x \in \in^{*} z$.

2. $x \sim y \rightarrow\left(x \in^{*} z \rightarrow y \in^{*} z\right)$.

3. $x \sim y \leftrightarrow(x=y)^{*}$.

4. $x \sim y \rightarrow\left(\varphi^{*}(x) \leftrightarrow \varphi^{*}(y)\right)$.

5. $\forall x \in \in^{*} z \varphi^{*} \leftrightarrow \forall x \in z \varphi^{*}$.

6. $\exists x \in^{*} z \varphi^{*} \leftrightarrow \exists x \in z \varphi^{*}$.

7. $x=y \rightarrow(x=y)^{*}$.

Proof. Clause 1 follows from the fact that $\sim$ is reflexive. Clause 2 follows from the definition of $\epsilon^{*}$, and 3 follows from 2 together with equivalences (5) and (6) above. Clause 4 is proved using induction on $\varphi$, with 3 as the base case. The forwards direction of 5 is easy, using 1 . For the other direction, suppose $\forall x \in z \varphi^{*}$ and $x \in^{*} z$. The latter means that there is an $x_{0} \in z$ such that $x_{0} \sim x$; but then $\varphi^{*}\left(x_{0}\right)$, and hence $\varphi^{*}(x)$ by 3 . The proof of 6 is similar to that of 5 . Clause 7 follows from 5,1 , and the definition of equality.

Clauses 5 and 6, together with Lemma 4.1, yield

CoROLLARY 4.7. The $*$-translation of any $\Delta_{0}$ formula is $\Delta_{1}$ in $K P^{\text {int }} \uparrow$.

LEMma 4.8. The $*$-translation of each axiom of $K P \mid$ is provable in $K P^{i n t} \uparrow$. The same is true for KP and KPint.

Proof. The fact that the $*$-translation of extensionality is provable in the intensional theory follows from clauses 2 and 3 of Lemma 4.6, and the $*$-translations of pairing and union follow from the corresponding axioms in the intensional theory, using clauses 1 and 5. Translations of instances of $\Delta_{0}$ separation and collection, as well as $\Sigma_{1}$ or full foundation, are handled using clauses 5 and 6 of Lemma 4.6, Corollary 4.7, and Lemma 4.1.

This yields

THEOREM 4.9. If $K P \uparrow$ proves a formula $\varphi$, then $K P^{\text {int }} \uparrow$ proves $\varphi^{*}$; and similarly for $K P$ and $K P^{\text {int }}$. 
In the next section we will consider versions of admissible set theory with an axiom of infinity; we can use Lemma 4.6 to show that Theorem 4.9 still holds with this addition. One may also wish to consider versions of Kripke-Platek set theory in which one has a set $\mathbb{N}$ containing the natural numbers as urelements, as well as the primitive recursive functions and a built-in notion of equality on that set. Once again, Theorem 4.9 still holds for these theories, provided that in the intensional versions we have the usual axioms governing equality on $\mathbb{N}$. The modifications necessary for this interpretation are well described in $[13,6]$, and pose no additional problems in the present setting.

§5. Interpreting intensional $K P$. Having dealt with extensionality, we can now restrict our attention to the interpretation of $K P^{\text {int }}$ and $K P^{\text {int }} \uparrow$. Let $I K P^{\text {int }}$ and $I K P^{i n t} \uparrow$ denote the corresponding theories where the underlying logic is intuitionistic. Our goal is to show that the classical theories are conservative over the intuitionistic ones for a certain class of formulae; for the moment, we will focus our attention on $K P^{\text {int }} \uparrow$. The argument below is modeled after the one in Section 3, but is more delicate because in $I K P^{\text {int }} \uparrow$ one can not, in general, prove the law of the excluded middle for $\Delta_{0}$ formulae. Nonetheless, we will again proceed in two steps, and make use of an intermediate theory based on intuitionistic logic. Many of the lemmata below are patterned after similar ones in [7].

In this setting it turns out that the primitive recursive relations of Section 3 are analogous to negative $\Delta_{0}$ formulae, and the $\Sigma_{1}$ formulae of arithmetic are analogous to what I will call "weak $\Sigma_{1}$ " formulae in the language of set theory. These are defined to be formulae of the form

$$
\exists w \neg \forall x \in w \varphi
$$

where $\varphi$ is negative and $\Delta_{0}$, and $w$ does not appear in $\varphi$. Being weak $\Sigma_{1}$ is more restrictive than being $\Sigma_{1}$; formula (7) does not quite assert that there is an $x$ satisfying $\neg \varphi$, but rather that there is a set $w$ of candidates, not all of which satisfy $\varphi$.

The intermediate theory $\left.I K P^{i n t \#}\right\rceil$ is defined to be the theory based on intuitionistic logic, given by the following axioms:

1. Pair and union: as in $K P$.

2. $\Delta_{0}$ separation $^{\#}$ : as in $K P$, except restricted to negative $\Delta_{0}$ formulae.

3. $\Delta_{0}$ collection ${ }^{\#}: \forall x \in z \exists y \varphi(x, y) \rightarrow \exists w \forall x \in z \neg \forall y \in w \neg \varphi(x, y)$ where $\varphi$ is $\Delta_{0}$ and negative.

4. $\Sigma_{1}$ foundation $\#$ : as in $K P$, except restricted to weak $\Sigma_{1}$ formulae.

Define the axiom schema

$$
\neg \forall x \varphi \rightarrow \exists w \neg \forall x \in w \varphi(x)
$$

where $\varphi$ is negative and $\Delta_{0}$. Since the converse direction is intuitionistically valid, $\left(M P_{\text {res }}\right)$ implies that the negation of any $\Pi_{1}$ formula is equivalent to something that is weak $\Sigma_{1}$.

In the first step we will use the double-negation translation to interpret $K P^{i n t} \uparrow$ in $I K P^{i n t \#} \uparrow+\left(M P_{\text {res }}\right)$. Since we no longer have the decidability of atomic formulae in the latter, here we must take $(y \in x)^{N}$ to be $\neg \neg y \in x$.

LemMa 5.1. Let $\varphi$ be any formula. Then the following are intuitionistically valid:

1. $y \in x \rightarrow \varphi^{N}$ iff $\neg \neg y \in x \rightarrow \varphi^{N}$. 
2. $(\forall y \in x \varphi)^{N}$ iff $\forall y \in x \varphi^{N}$.

3. $(\exists y \in x \varphi)^{N}$ iff $\neg \forall y \in x \neg \varphi^{N}$.

Hence, the double-negation translation of any $\Delta_{0}$ formula is intuitionistically equivalent to a $\Delta_{0}$ formula.

Proof. The last claim is proved by induction on formulae, using equivalences 2 and 3.

The right-to-left direction of 1 follows from the fact that $y \in x$ implies $\neg \neg y \in x$ intuitionistically. Conversely, $y \in x \rightarrow \varphi^{N}$ implies $\neg \varphi^{N} \rightarrow \neg y \in x$, and hence $\neg \neg y \in x \rightarrow \neg \neg \varphi^{N}$. But since $\neg \neg \varphi \rightarrow \varphi$ is classically valid, $\neg \neg \varphi^{N}$ implies $\varphi^{N}$ intuitionistically.

Regarding 2, we have

$$
\begin{aligned}
(\forall y \in x \varphi)^{N} & \equiv \forall y(y \in x \rightarrow \varphi)^{N} \\
& \equiv \forall y\left(\neg \neg y \in x \rightarrow \varphi^{N}\right)
\end{aligned}
$$

which is equivalent to $\forall y \in x \varphi^{N}$ by part 1 . Clause 3 is proved by noting that $\exists y \in x \varphi$ and $\neg \forall y \in x \neg \varphi$ are classically equivalent, and applying 2 with $\neg \varphi$ in place of $\varphi$.

LEMMA 5.2. IKPint\# $\uparrow+\left(M P_{\text {res }}\right)$ proves the double-negation translation of each axiom of $K P^{\text {int }} \uparrow$.

Proof. Pair and union imply their double-negation translations, and the doublenegation translation of any instance of $\Delta_{0}$ separation is implied by an instance of $\Delta_{0}$ separation $^{\#}$.

To handle $\Delta_{0}$ collection, note that by Lemma 5.1 its double-negation translation is intuitionistically equivalent to

$$
\forall x \in z \neg \forall y \neg \varphi^{N}(x, y) \rightarrow \neg \forall w \neg \forall x \in z \neg \forall y \in w \neg \varphi^{N}(x, y) .
$$

Arguing in $I K P^{i n t \#} \uparrow+\left(M P_{\text {res }}\right)$, suppose the antecedent is true. By $\left(M P_{\text {res }}\right)$ we have

$$
\forall x \in z \exists s \neg \forall y \in s \neg \varphi^{N}(x, y) .
$$

By $\Delta_{0}$ collection ${ }^{\#}$ we have

$$
\exists w_{1} \forall x \in z \neg \forall s \in w_{1} \neg \neg \forall y \in s \neg \varphi^{N}(x, y) .
$$

Given such a set $w_{1}$, let $w$ be the set containing $\bigcup w_{1}$ asserted to exist by the union axiom. Then for every $x$ in $z$ we have

$$
\forall y \in w \neg \varphi^{N}(x, y) \rightarrow \forall s \in w_{1} \forall y \in s \neg \varphi^{N}(x, y) ;
$$

weakening the conclusion and taking the contrapositive yields

$$
\neg \forall s \in w_{1} \neg \neg \forall y \in s \neg \varphi^{N}(x, y) \rightarrow \neg \forall y \in w \neg \varphi^{N}(x, y) .
$$

Combining this with (9) yields

$$
\exists w \forall x \in z \neg \forall y \in w \neg \varphi^{N}(x, y),
$$

which implies the conclusion of (8).

Finally, since $\left(M P_{\text {res }}\right)$ implies that the double-negation of a $\Sigma_{1}$ formula is weak $\Sigma_{1}$, the double-negation of an instance of $\Sigma_{1}$ foundation is equivalent to an instance of $\Sigma_{1}$ foundation ${ }^{\#}$. 
Let $\mathscr{C}_{\text {res }}$ be the smallest set that is closed under conjunction, implication, and universal quantification, and that contains all weak $\Sigma_{1}$ formulae in which every atomic subformula is preceeded by at least one negation. One can show inductively that for each formula $\varphi$ in $\mathscr{C}_{\text {res }}, I K P^{i n t \#} \uparrow+\left(M P_{\text {res }}\right)$ proves that $\varphi$ is equivalent to $\varphi^{N}$. Thus we have

THEOREM 5.3. K $K P^{\text {int }} \uparrow$ is conservative over IKP $P^{\text {int }} \uparrow+\left(M P_{\text {res }}\right)$ for formulae in $\mathscr{C}_{\text {res }}$.

We are now ready to use the forcing framework of Section 2 to reduce $I K P^{\text {int } \# \uparrow} \uparrow+$ $\left(M P_{\text {res }}\right)$ to $I K P^{\text {int }} \uparrow$. Here the appropriate conditions are finite sets of $\Pi_{1}$ sentences with parameters. Using the third method described in Section 3, let $\operatorname{Tr}_{r e s}^{m}$ be a $\Delta_{0}$ truth predicate for sufficiently many $\Delta_{0}$ sentences with parameters, and if $p$ is a set of $\Pi_{1}$ formulae $\left\{\forall x \varphi_{1}(x), \ldots, \forall x \varphi_{k}(x)\right\}$, let

$$
\operatorname{Tr}(p, u) \equiv \bigwedge_{i=1}^{k} \forall x \in u \varphi_{i}(x),
$$

where $\operatorname{Tr}_{r e s}^{m}$ is used to express the right-hand side. For any formula $\varphi$, define

$$
p \vdash \varphi \equiv \exists u(\operatorname{Tr}(p, u) \rightarrow \varphi) .
$$

Intuitively, $u$ provides a "proof" of $\varphi$ from $p$ by giving a bound on the universal quantifiers that is sufficiently large to witness the fact that $\varphi$ follows from the formulae in $p$.

LEMMA 5.4. For any formula $\varphi$, the following is provable in IKP int $\uparrow:$ If $u \subseteq v$, then

1. $\operatorname{Tr}(p, v)$ implies $\operatorname{Tr}(p, u)$, and

2. $\operatorname{Tr}(p, u) \rightarrow \varphi$ implies $\operatorname{Tr}(p, v) \rightarrow \varphi$.

LeMma 5.5. Let $\varphi$ and $\psi$ be any formulae, and let $\theta$ be any $\Delta_{0}$ formula. Then the following are provable in IKP $P^{\text {int }} \uparrow$ :

1. If $p \vdash \varphi$ and $q \supseteq p$ then $q \vdash \varphi$.

2. $\theta \vdash \theta$.

3. $p \vdash(\varphi \wedge \psi)$ iff $p \vdash \varphi$ and $p \vdash \psi$.

4. $p \vdash(\theta \rightarrow \psi)$ iff $p, \theta \vdash \psi$.

5. If $p \vdash(\varphi \rightarrow \psi)$ and $q \vdash \varphi$ then $p, q \vdash \psi$.

6. The following are equivalent:

(a) $p \vdash \forall x \in z \theta$.

(b) $\forall x p \vdash(x \in z \rightarrow \theta)$.

(c) $\forall x \in z p \vdash \theta$.

Proof. Clauses 1 and 2 follow from the definition of $\operatorname{Tr}(p, u)$ and the adequacy of $\operatorname{Tr}_{r e s}^{m}$.

For the forward direction of 3 , if $\operatorname{Tr}(p, u) \rightarrow \varphi \wedge \psi$ then $\operatorname{Tr}(p, u) \rightarrow \varphi$ and $\operatorname{Tr}(p, u) \rightarrow \psi$. Conversely, if $\operatorname{Tr}(p, u) \rightarrow \varphi$ and $\operatorname{Tr}(p, v) \rightarrow \psi$ then $\operatorname{Tr}(p, u \cup v) \rightarrow$ $\varphi \wedge \psi$ by Lemma 5.4 .

For 4, we have

$$
\begin{array}{lll}
\operatorname{Tr}(p, u) \rightarrow(\theta \rightarrow \psi) & \text { iff } & \operatorname{Tr}(p, u) \wedge \theta \rightarrow \psi \\
& \text { iff } \operatorname{Tr}(p \cup\{\theta\}, u) \rightarrow \psi .
\end{array}
$$

Regarding 5, if $\operatorname{Tr}(p, u) \rightarrow(\varphi \rightarrow \psi)$ and $\operatorname{Tr}(q, v) \rightarrow \varphi$, then, using the monotonicity of $\operatorname{Tr}$ in both arguments, we have $\operatorname{Tr}(p \cup q, u \cup v) \rightarrow \psi$. 
Finally, regarding 6, (a) implies (b) intuitionistically, and (b) implies (c) intuitionistically as well. To show that (c) implies (a), suppose

$$
\forall x \in z \exists u(\operatorname{Tr}(p, u) \rightarrow \theta) .
$$

Using $\Delta_{0}$ collection, we have

$$
\exists v_{1} \forall x \in z \exists u \in v_{1}(\operatorname{Tr}(p, u) \rightarrow \theta) .
$$

Given such a set $v_{1}$ we can let $v$ be $\bigcup v_{1}$, in which case $u \in v_{1}$ implies $u \subseteq v$; by Lemma 5.4 this shows

$$
\exists v \forall x \in z(\operatorname{Tr}(p, v) \rightarrow \theta),
$$

which is equivalent to (a).

When $\theta$ is atomic, define $p \Vdash \theta$ to be $p \vdash \theta$, and extend the forcing relation to arbitrary formulae in the language of set theory according to the clauses in Section 2.

Lemma 5.6. Let $\varphi$ be any negative $\Delta_{0}$ formula. Then in IKP ${ }^{\text {int }} \uparrow$ one can prove

$$
p \Vdash \varphi \text { iff } p \vdash \varphi .
$$
do.

Proof. By induction on the complexity of $\varphi$. If $\varphi$ is atomic, there is nothing to

If $\varphi$ is of the form $\theta \wedge \psi$, apply the inductive hypothesis and Lemma 5.5.3.

When $\varphi$ is of the form $\theta \rightarrow \psi$, we have

$$
\begin{aligned}
p \Vdash(\theta \rightarrow \psi) & \text { iff } \quad \forall q(q \Vdash \theta \rightarrow p, q \Vdash \psi) \\
& \text { iff } \quad \forall q(q \vdash \theta \rightarrow p, q \vdash \psi) .
\end{aligned}
$$

We need to show that the last line is equivalent to $p \vdash(\theta \rightarrow \psi)$. In the forwards direction, if we take $q$ to be $\{\theta\}$, we have $p, \theta \vdash \psi$ and hence $p \vdash(\theta \rightarrow \psi)$ by Lemma 5.5.4. Conversely, assuming $p \vdash(\theta \rightarrow \psi)$, Lemma 5.5.5 tells us that if $q$ is any condition and $q \vdash \theta$, then $p, q \vdash \psi$.

Finally, suppose $\varphi$ is of the form $\forall x \in z \theta$. Then we have

$$
\begin{array}{ll}
p \Vdash \forall x \in z \theta & \text { iff } \quad \forall x p \Vdash(x \in z \rightarrow \theta) \\
& \text { iff } \quad \forall x p \vdash(x \in z \rightarrow \theta) .
\end{array}
$$

By Lemma 5.5.6 this is equivalent to $p \vdash \forall x \in z \theta$.

We are almost ready to verify that the axioms of the intermediate theory $I K P^{\text {int } t \#}\lceil+$ $\left(M P_{\text {res }}\right)$ are forced in $I K P^{i n t} \uparrow$. The following lemma incorporates many of the technical details.

LeMma 5.7. Let $\varphi$ be any negative $\Delta_{0}$ formula, let be $\psi$ be any weak $\Sigma_{1}$ formula, and let $\eta$ be arbitrary. Then the following are provable in IKPint $\uparrow$ :

1. $\Vdash \exists x \varphi$ iff $\exists x \varphi$.

2. $\forall x \varphi \Vdash \forall x \varphi$.

3. If $p \Vdash \neg \forall x \varphi$ then $p \Vdash \exists w \neg \forall x \in w \varphi$.

4. If $p \Vdash \forall x \in y \eta$ then $\forall x \in y p \Vdash \eta$.

5. If $p \Vdash \forall x \in y \exists z \varphi$ then $p \Vdash \exists w \forall x \in y \neg \forall z \in w \neg \varphi$.

6. $p \Vdash \forall x \in y \psi$ iff $\forall x \in y p \Vdash \psi$. 
Proof. Clause 1 follows from the definition of $\Vdash$ and Lemma 5.6.

Regarding 2, we have

$$
\begin{aligned}
\forall x \varphi(x) \Vdash \forall x \varphi(x) \quad \text { iff } \quad \forall z(\forall x \varphi(x) \Vdash \varphi(z)) \\
\text { iff } \quad \forall z(\forall x \varphi(x) \vdash \varphi(z)) \\
\text { iff } \quad \forall z \exists u\left(\operatorname{Tr}_{r e s}^{m}(\ulcorner\forall x \in u \varphi(x)\urcorner) \rightarrow \varphi(z)\right) .
\end{aligned}
$$

Given $z$, take $u=\{z\}$.

Regarding 3, suppose $p \Vdash \neg \forall x \varphi$. Then

$$
\forall q(q \Vdash \forall x \varphi \rightarrow p, q \Vdash \perp) .
$$

In particular, from 2 we have $p, \forall x \varphi \Vdash \perp$; that is,

$$
\exists u(\operatorname{Tr}(p, u) \wedge \forall x \in u \varphi \rightarrow \perp)
$$

and therefore

$$
\exists u(\operatorname{Tr}(p, u) \rightarrow \neg \forall x \in u \varphi) .
$$

Taking $w=u$ we have

$$
\exists w p \vdash \neg \forall x \in w \varphi .
$$

By Lemma 5.6 and the definition of $\Vdash$, this is equivalent to

$$
p \Vdash \exists w \neg \forall x \in w \varphi .
$$

Regarding 4, suppose $p \Vdash \forall x \in y \eta$. Then

$$
\forall x, q(q \Vdash x \in y \rightarrow p, q \Vdash \eta) .
$$

In particular, if $x \in y$ then $\Vdash x \in y$, and hence $p \Vdash \eta$.

It should be no surprise that proving 5 requires the use of $\Delta_{0}$ collection. Suppose $p \Vdash \forall x \in z \exists y \varphi(x, y)$, where $\varphi$ is $\Delta_{0}$ and negative; we need to show $p \Vdash \exists w \forall x \in$ $z \neg \forall y \in w \neg \varphi(x, y)$. By 4 and the definition of $\Vdash$, the assumption implies

$$
\forall x \in z \exists y p \Vdash \varphi(x, y)
$$

which is equivalent to

$$
\forall x \in z \exists y p \vdash \varphi(x, y)
$$

by Lemma 5.6. This is, by definition,

$$
\forall x \in z \exists y, u(\operatorname{Tr}(p, u) \rightarrow \varphi(x, y)) .
$$

Pairing $y$ and $u$ and using $\Delta_{0}$ collection, we get

$$
\exists w, v_{1} \forall x \in z \exists y \in w, u \in v_{1}(\operatorname{Tr}(p, u) \rightarrow \varphi(x, y)) .
$$

Letting $v$ be $\bigcup v_{1}$ we have

$$
\exists w, v \forall x \in z \exists y \in w(\operatorname{Tr}(p, v) \rightarrow \varphi(x, y)),
$$

which intuitionistically implies

$$
\exists w, v \forall x \in z(\operatorname{Tr}(p, v) \rightarrow \exists y \in w \varphi(x, y))
$$

and hence

$$
\exists w, v \forall x \in z(\operatorname{Tr}(p, v) \rightarrow \neg \forall y \in w \neg \varphi(x, y)) .
$$

This last formula is intuitionistically equivalent to

$$
\exists w, v(\operatorname{Tr}(p, v) \rightarrow \forall x \in z \neg \forall y \in w \neg \varphi(x, y)),
$$


which is, by definition,

$$
\exists w p \vdash \forall x \in z \neg \forall y \in w \neg \varphi(x, y) .
$$

By Lemma 5.6 and the definition of $\Vdash$, this is equivalent to

$$
p \Vdash \exists w \forall x \in z \neg \forall y \in w \neg \varphi(x, y),
$$

as desired.

Finally, regarding 6 , note that 4 takes care of the forwards direction. For the converse direction, suppose

$$
\forall x \in y p \Vdash \exists z \neg \forall v \in z \varphi
$$

where $\varphi$ is $\Delta_{0}$ and negative. Then by the definition of $\Vdash$ and Lemma 5.6 we have

$$
\forall x \in y \exists z p \vdash \neg \forall v \in z \varphi .
$$

Since $p \vdash \neg \forall v \in z \varphi$ is $\Sigma_{1}$, we can pair existential quantifiers and use $\Delta_{0}$ collection to obtain

$$
\exists s_{1} \forall x \in y \exists z \in s_{1} p \vdash \neg \forall v \in z \varphi .
$$

If $s$ is $\bigcup s_{1}$, then $z \in s_{1}$ implies $z \subseteq s$ and so $\neg \forall v \in z \varphi$ implies $\neg \forall v \in s \varphi$. As a result, the last formula implies

$$
\exists s \forall x \in y p \vdash \neg \forall v \in s \varphi .
$$

By Lemma 5.5.6, this is equivalent to

$$
\exists s p \vdash \forall x \in y \neg \forall v \in s \varphi
$$

and hence

$$
p \Vdash \exists s \forall x \in y \neg \forall v \in s \varphi .
$$

Since intuitionistic validities are forced, this implies

$$
p \Vdash \forall x \in y \exists s \neg \forall v \in s \varphi
$$

as desired.

LEMMA 5.8. IKP $P^{\text {int }} \uparrow$ proves that each axiom of IKP int\# $\uparrow+\left(M P_{\text {res }}\right)$ is forced.

PRoof. If $\varphi$ is pair, union, or $\Delta_{0}$ separation $^{\#}$, then by Lemma 5.7.1 $\Vdash \varphi$ is equivalent to $\varphi$, which follows from the corresponding axiom of $I K P^{\text {int }} \uparrow$.

Lemma 5.7.3 implies that $\left(M P_{\text {res }}\right)$ is forced, and Lemma 5.7.5 takes care of $\Delta_{0}$ collection $^{\#}$. Finally, to handle $\Sigma_{1}$ foundation $^{\#}$, suppose

$$
p \Vdash \forall x(\forall y \in x \psi(y) \rightarrow \psi(x)),
$$

where $\psi$ is weak $\Sigma_{1}$. By definition this means

$$
\forall x, q(q \Vdash \forall y \in x \psi(y) \rightarrow p, q \Vdash \psi(x)) .
$$

In particular, taking $q$ to be $p$ and applying Lemma 5.7.6, we have that for every $x$

$$
\forall y \in x p \Vdash \psi(y) \text { implies } p \Vdash \psi(x) .
$$

Since $p \Vdash \psi(y)$ is equivalent to a $\Sigma_{1}$ formula, we can use $\Sigma_{1}$ foundation in the target theory to show

$$
\forall x p \Vdash \psi(x)
$$

and hence $p \Vdash \forall x \psi(x)$, as desired. 
Let $\mathscr{D}_{\text {res }}$ be the smallest set that contains the negative $\Delta_{0}$ formulae and is closed under conjunction, disjunction, and universal and existential quantification. For every $\varphi$ in $\mathscr{D}_{\text {res }}, I K P^{\text {int }} \uparrow$ proves that $\Vdash \varphi$ is equivalent to $\varphi$. As a result, we have

THEOREM 5.9. IKP $P^{\text {int } \# \uparrow} \uparrow+\left(M P_{\text {res }}\right)$ is conservative over IKP ${ }^{\text {int }} \uparrow$ for formulae in $\mathscr{D}_{\text {res }}$.

Taken together, Theorems 5.3 and 5.9 yield

THEOREM 5.10. Suppose KPint $\uparrow$ proves $\forall x \exists y \varphi(x, y)$, where $\varphi$ is $\Delta_{0}$. Then IKPint $\uparrow$ proves $\forall x \exists w \neg \forall y \in w \neg \varphi^{N}(x, y)$.

Proof. Classically the two formulae are equivalent, and the latter is in $\mathscr{C}_{\text {res }} \cap$ $\mathscr{D}_{\text {res. }}$.

Combining this with Theorem 4.9 yields a reduction of the extensional, classical theory to the intensional, intuitionistic one:

THeOREM 5.11. Suppose KP $\left\lceil\right.$ proves $\forall x \exists y \varphi(x, y)$, where $\varphi$ is $\Delta_{0}$. Then IKPint $\uparrow$ proves $\forall x \exists w \neg \forall y \in w \neg \varphi^{* N}(x, y)$.

Proof. By Corollary 4.7, $\varphi^{*}(x, y)$ is $\Delta$ in $K P^{i n t} \uparrow$, and so equivalent to a $\Sigma_{1}$ formula $\exists u \theta(x, y, u)$. Pairing quantifiers and applying the previous theorem, we have that IKP $P^{\text {int }} \uparrow$ proves $\forall x \exists w \neg \forall y \in w, u \in w \neg \theta^{N}(x, y, u)$. The conclusion follows from the fact that $\neg \forall u \in w \neg \theta^{N}(x, y, u)$ implies $\varphi^{* N}(x, y)$.

What about adding the full foundation schema to both sides? Interpreting the $\Sigma_{1}$ foundation axiom of $I K P^{\text {int } I \#} \mid$ made use of Lemma 5.7.6, which asserts that $p \Vdash \forall x \in y \eta$ is equivalent to $\forall x \in y p \Vdash \eta$ when $\eta$ is weak $\Sigma_{1}$. To interpret $K P^{i n t}$, it

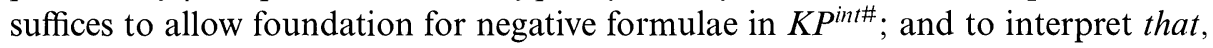
we need to know that the equivalence given by Lemma 5.7.6 holds for arbitrary negative formulae. This fact is supplied by the following two lemmata.

Lemma 5.12. Suppose $\varphi$ and $\psi$ are any two $\Delta_{0}$ formulae. Then IKPint $\uparrow$ proves

$$
(\varphi \rightarrow \exists u \psi) \text { implies } \exists v(\varphi \rightarrow \exists u \in v \psi)
$$

where $v$ is any new variable.

Proof. Arguing in $I K P^{\text {int }} \uparrow$, suppose $\varphi \rightarrow \exists u \psi$. We can use pairing and $\Delta_{0}$ separation to prove the existence of $\{\emptyset\}$, and then the existence of a set $z$ such that

$$
\forall w(w \in z \leftrightarrow w \in\{\emptyset\} \wedge \varphi) .
$$

If there is any $w$ in $z$, then $\varphi$ holds, and hence so does $\exists u \psi$. In other words, we have

$$
\forall w \in z \exists u \psi \text {. }
$$

By $\Delta_{0}$ collection we have

$$
\exists v \forall w \in z \exists u \in v \psi .
$$

But if $\varphi$ holds then $\emptyset$ is in $z$, so we have

$$
\exists v(\varphi \rightarrow \exists u \in v \psi)
$$

as needed.

LeMMa 5.13. If $\varphi$ is negative, IKP ${ }^{\text {int }} \uparrow$ proves

$$
(x \in y \rightarrow p \Vdash \varphi) \quad \text { iff } \quad p \Vdash(x \in y \rightarrow \varphi)
$$

and hence

$$
\forall x \in y p \Vdash \varphi \quad \text { iff } \quad p \Vdash \forall x \in y \varphi .
$$


PROof. The second claim follows easily from the first, and proving the right-toleft direction of the first claim is straightforward, as in the proof of Lemma 5.7.4. The left-to-right direction of the first claim is proved by induction on the complexity of $\varphi$.

In the atomic case, suppose $x \in y \rightarrow p \Vdash \theta$. Then we have

$$
x \in y \rightarrow p \vdash \theta
$$

and hence

$$
x \in y \rightarrow \exists u(\operatorname{Tr}(p, u) \rightarrow \theta) .
$$

By the previous lemma, there is a $v_{1}$ such that

$$
x \in y \rightarrow \exists u \in v_{1}(\operatorname{Tr}(p, u) \rightarrow \theta) .
$$

Letting $v$ be $\bigcup v_{1}$, we have

$$
x \in y \rightarrow(\operatorname{Tr}(p, v) \rightarrow \theta) .
$$

Rearranging the antecedents in this last formula yields

$$
\exists v(\operatorname{Tr}(p, v) \rightarrow(x \in y \rightarrow \theta)),
$$

which is the same as

$$
p \vdash(x \in y \rightarrow \theta) .
$$

By Lemma 5.6, this is equivalent to $p \Vdash(x \in y \rightarrow \theta)$.

Handling the cases involving the connectives $\wedge, \rightarrow$, and $\forall$ is straightforward, using the following intuitionistic equivalences:

- $(\eta \rightarrow(\varphi \wedge \psi)) \leftrightarrow((\eta \rightarrow \varphi) \wedge(\eta \rightarrow \psi))$.

- $(\eta \rightarrow(\varphi \rightarrow \psi)) \leftrightarrow((\eta \rightarrow \varphi) \rightarrow(\eta \rightarrow \psi))$.

- $(\eta \rightarrow \forall x \varphi) \leftrightarrow \forall x(\eta \rightarrow \varphi)$, if $x$ is not free in $\eta$.

This completes the proof.

Since we can now interpret foundation for negative formulae as in the proof of Lemma 5.8, we have

THEOREM 5.14. KP is interpretable in IKP int.

Here and for the rest of this section, I will take the wording of Theorem 5.14 as an abbreviation for the assertion that Theorem 5.11 still holds when one replaces $K P \nmid$ and $I K P^{i n t} \uparrow$ by the theories mentioned.

We can generalize these results. Suppose $\varphi, \psi, \theta$, and $\eta$ are formulae such that

- $K P^{i n t} \uparrow+\psi$ proves $\varphi^{*}$,

- $I K P^{i n t \#} \uparrow+\left(M P_{r e s}\right)+\theta$ proves $\psi^{N}$, and

- $I K P^{i n t} \uparrow+\eta$ proves $\Vdash \theta$.

Then $K P \uparrow+\varphi$ is interpretable in $I K P^{i n t} \uparrow+\eta$. For example, suppose we take the axiom of infinity to be given (as in [3]) by

(infinity)

$$
\exists x(\exists y \in x \operatorname{zero}(y) \wedge \forall y \in x \exists z \in x \operatorname{succ}(y, z)),
$$

where zero $(y)$ is the formula $\forall z \in y \perp$ and $\operatorname{succ}(y, z)$ is

$$
y \in z \wedge \forall u \in y(u \in z) \wedge \forall u \in z(u \in y \vee u=y) .
$$


Then clauses 1 and 7 of Lemma 4.6 guarantee that (infinity*) follows from (infinity), and the double-negation translation of (infinity) is intuitionistically implied by

$$
\exists x\left(\neg \forall y \in x \neg \operatorname{zero}^{N}(y) \wedge \forall y \in x \neg \forall z \in x \neg \operatorname{succ}^{N}(y, z)\right) .
$$

Since $(10)$ is in $\mathscr{D}_{\text {res }}$, IKP $P^{\text {int }} \uparrow$ proves that it is forced if and only if it is true. Furthermore, since (10) is implied by (infinity), we have

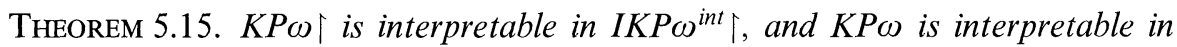
IKP $\omega^{\text {int }}$.

Here the symbol $\omega$ in a theory's name indicates that infinity is to be included among the axioms.

By analogous reasoning we have, for example,

THEOREM 5.16. Let $\theta(x, y)$ be any $\Delta_{0}$ formula. Then $K P \omega+\forall x \exists y \theta(x, y)$ is interpretable in $I K P \omega^{\text {int }}+\forall x \exists y \theta^{* N}(x, y)$.

Suppose that instead of adding an axiom of infinity, we take $K P u$ to be the theory of [16], with a set $\mathbb{N}$ of natural numbers as urelements. Then, according to the discussion at the end of Section 4, KPu is interpreted in $I K P u^{i n t}$. As a result, if $K P u$ proves $\forall x \in \mathbb{N} \exists y \in \mathbb{N} A(x, y)$ for some primitive recursive predicate $A$, then $I K P u^{\text {int }}$ proves $\forall x \in \mathbb{N} \neg \forall y \in \mathbb{N} \neg A(x, y)$. Since the latter theory is closed under the Friedman-Dragalin translation (see [14]), we have

THEOREM 5.17. KPu is conservative over IKPu $u^{\text {int }}$ for $\Pi_{2}$ sentences of arithmetic.

In [16], Jäger also considers theories in which one drops the foundation axiom and replaces it with various forms of induction over the natural numbers. The methods discussed in this section apply to these theories as well: the analogues of Theorem 5.10 hold for the intensional versions of $K P u^{0}+\left(\Sigma_{1}\right.$ induction $)$ and $K P u^{0}+($ induction $)$. However, the corresponding version of Theorem 5.11 does not follow, since the interpretation of extensionality in Section 4 requires $\Sigma_{1}$ foundation.

$\S 6$. Subsystems of second-order arithmetic. The theory $K P u^{0}+($ induction $)$, discussed at the end of the previous section, has the same strength as the subsystem of second-order arithmetic, $\Sigma_{I}^{I}-A C$ (see [16]). In this section I will show that the methods we have been using can be applied to $\Sigma_{l}^{I}$ - $A C$ directly, reducing it, as well, to its intuitionistic counterpart. ${ }^{4}$ Here the task is somewhat easier than that of interpreting $K P$, for two reasons: we do not have to worry about extensionality, and induction is easier to interpret than foundation.

The language of second-order arithmetic is two-sorted, extending the language of first-order arithmetic with variables $X, Y, Z, \ldots$ ranging over sets of natural numbers, and a relation $\in$ between terms of the two sorts. Equality between second-order objects is taken to be defined in terms of first-order equality, so that $X=Y$ is given by $\forall z(z \in X \leftrightarrow z \in Y)$. A formula is said to be arithmetic if it contains no

\footnotetext{
${ }^{4}$ The proof-theoretic equivalence of $\Sigma_{l}^{l}-A C$ and $\Sigma_{I}^{l}-A C^{i}$ is due to Aczel [1]; it can also be obtained via ordinal analysis. The methods described in this section can also be used to interpret the stronger theory $\Sigma_{1}^{1}-D C$ and the weaker theory $\Delta_{1}^{1}-C A$ in their intuitionistic counterparts. Since Friedman [12] has shown that one can interpret both $\Sigma_{1}^{1}-D C$ and $\Sigma_{1}^{1}-A C$ in $\Delta_{1}^{1}-C A$, the analysis here shows that the proof-theoretic strength of all these theories, taken in either the classical or intuitionistic versions, are the same.
} 
second-order quantifiers, though it may contain second-order variables; it is said to be $\Sigma_{1}^{l}$ if it is either arithmetic or of the form $\exists Y \varphi$, where $\varphi$ is arithmetic.

If we let $\langle\cdot, \cdot\rangle$ denote a primitive recursive pairing function on the natural numbers and read $t \in Y_{x}$ as $\langle t, x\rangle \in Y$, we can think of the set $Y$ as coding a countable sequence of sets indexed by $x$. We can also interpret a single set $Y$ as coding a countable collection of sets, and introduce bounded second-order quantification by

$$
\begin{aligned}
& \forall X \in Y \varphi(X) \equiv \forall x \varphi\left(Y_{x}\right) \\
& \exists X \in Y \varphi(X) \equiv \exists x \varphi\left(Y_{x}\right)
\end{aligned}
$$

While this device is suggestive, one should keep in mind that in this context that apparent second-order quantifiers are, in reality, first-order.

The axioms of $\Sigma_{I}^{I}-A C$ are as follows:

1. Quantifier-free defining equations for the first-order symbols of arithmetic.

2. Arithmetic comprehension $(A C A): \exists Y \forall x(x \in Y \leftrightarrow \varphi(x))$, where $\varphi$ is arithmetic and $Y$ does not appear in $\varphi$.

3. Arithmetic choice $\left(\Sigma_{I}^{I}-A C\right): \forall x \exists Y \varphi(x, Y) \rightarrow \exists Y \forall x \varphi\left(x, Y_{x}\right)$, where $\varphi$ is arithmetic.

4. Induction for arbitrary formulae in the language.

By coding pairs of sets as a single set, one can easily extend the choice principle to $\Sigma_{1}^{1}$ formulae, which explains the name. $\Sigma_{l}^{I}-A C_{0}$ denotes the theory in which induction is restricted to sets of natural numbers; in the presence of arithmetic comprehension, this set induction axiom implies the schema of induction for arbitrary arithmetic formulae. $\Sigma_{I}^{l}-A C^{i}$ and $\Sigma_{I}^{l}-A C_{0}^{i}$ denote the corresponding intuitionistic theories.

The following lemma makes the analogy to $K P$ more salient.

LEMMA 6.1. Over the other axioms of $\Sigma_{l}^{l}-A C_{0},\left(\Sigma_{l}^{l}-A C\right)$ is equivalent to $\left(\Sigma_{I}^{l}-A C^{\prime}\right)$

$$
\forall x \exists Y \varphi(x, Y) \rightarrow \exists W \forall x \exists Y \in W \varphi(x, Y) .
$$

Proof. The conclusion of $\left(\Sigma_{I}^{l}-A C\right)$ clearly implies the conclusion of $\left(\Sigma_{I}^{l}-A C^{\prime}\right)$ : if $\forall x \varphi\left(x, Y_{x}\right)$, we can just take $W$ to be $Y$.

Conversely, suppose $\forall x \exists Y \in W \varphi(x, Y)$; that is, $\forall x \exists y \varphi\left(x, W_{y}\right)$. By arithmetic induction, we can show that for every $x$ there is a least number $u_{x}$ satisfying $\varphi\left(x, W_{u_{*}}\right)$. Using arithmetic comprehension to set

$$
Y=\left\{\langle x, v\rangle \mid v \in W_{u_{*}}\right\},
$$

we have $\forall x \varphi\left(x, Y_{x}\right)$, as desired.

If $U$ is construed as a countable collection of countable collections of sets, let $\bigcup U$ be given by

$$
\{\langle x,\langle y, z\rangle\rangle \mid\langle\langle x, y\rangle, z\rangle \in U\} .
$$

If we define the subset relation for countable collections of sets by

$$
X \sqsubseteq Y \equiv \forall w \exists z\left(X_{w}=Y_{z}\right),
$$

we then have that for every $x, U_{x} \sqsubseteq \bigcup U$.

Having seen the developments of the previous section, the reader can anticipate the translation to the current setting. First we need to define an intermediate theory $\Sigma_{I}^{l}-A C^{\#}$, in which negative arithmetic formulae replace the negative $\Delta_{0}$ formulae of Section 5. The appropriate axioms are: 
1. The quantifier-free defining equations for the first-order symbols of arithmetic.

2. $\left(A C A^{\#}\right): \exists Y \forall x(x \in Y \leftrightarrow \varphi(x))$, where $\varphi$ is negative and arithmetic and $Y$ does not occur in $\varphi$.

3. $\left(\Sigma_{l}^{l}-A C^{\#}\right): \forall x \exists Y \varphi(x, Y) \rightarrow \exists Y \forall x \varphi\left(x, Y_{x}\right)$, where $\varphi$ is negative arithmetic.

4. Induction for arbitrary formulae.

Let $\Sigma_{l}^{l}-A C_{0}^{\#}+\left(\Sigma_{l}^{l}\right.$ induction $\left.{ }^{\#}\right)$ denote the theory in which induction is restricted to formulae of the form $\exists Y \varphi$, where $\varphi$ is arithmetic and negative. As in Section 5, I will say that a formula is weak $\Sigma_{1}^{1}$ if it is of the form $\exists W \neg \forall X \in W \varphi$, where $\varphi$ is arithmetic and negative and $W$ is not free in $\varphi$. Thanks to the fact that induction is easier to interpret than foundation, note that here we do not have to restrict $\Sigma_{1}^{1}$ induction $^{\#}$ to weak $\Sigma_{1}^{1}$ formulae.

Finally, let us define

$$
\left(M P_{\text {crith }}\right) \quad \neg \forall X \varphi \rightarrow \exists W \neg \forall X \in W \varphi
$$

for $\varphi$ arithmetic and negative. As expected we have

Lemma 6.2. $\Sigma_{l}^{l}-A C_{0}^{\#}+\left(\Sigma_{l}^{l}\right.$ induction $\left.^{\#}\right)$ proves the double negation of every axiom of $\Sigma_{l}^{l}-A C_{0}+\left(\Sigma_{l}^{l}\right.$ induction $)$; similarly for $\Sigma_{l}^{l}-A C^{\#}$ and $\Sigma_{l}^{l}-A C$.

Proof. The double-negations of the quantifier-free defining equations are equivalent to themselves, and the double-negation of $(A C A)$ is implied by $\left(A C A^{\#}\right)$. Using $\left(M P_{\text {arith }}\right)$, the double-negation of a $\Sigma_{1}^{1}$ formula is weak $\Sigma_{1}^{1}$, so each instance of $\Sigma_{1}^{1}$ induction translates to an instance of $\Sigma_{1}^{1}$ induction"; and, of course, the arbitrary schema of induction in $\Sigma_{l}^{l}-A C$ translates to induction in $\Sigma_{l}^{l}-A C^{\#}$.

Finally, consider arithmetic choice. By Lemma 6.1, it suffices to interpret the $\mathrm{N}$-translation of $\left(\Sigma_{l}^{l}-A C^{\prime}\right)$. The hypothesis translates to

$$
\forall x \neg \forall Y \neg \varphi^{N}(x, Y),
$$

which, by $\left(M P_{\text {arith }}\right)$, is equivalent to

$$
\forall x \exists S \neg \forall Y \in S \neg \varphi^{N}(x, Y) .
$$

Applying $\left(\Sigma_{l}^{l}-A C^{\#}\right)$ yields

$$
\exists S \forall x \neg \forall Y \in S_{x} \neg \varphi^{N}(x, Y) .
$$

Setting $W$ to $\bigcup S$ we have

$$
\exists W \forall x \neg \forall Y \in W \neg \varphi^{N}(x, Y),
$$

which implies the translation of the conclusion of $\left(\Sigma_{l}^{l}-A C^{\prime}\right)$.

Let $\mathscr{C}_{\text {arith }}$ be the smallest class of formulae that is closed under conjunction, implication, and universal quantification, and that contains all the weak $\Sigma_{1}^{1}$ formulae in which every subformula of the form $t \in X$ is preceeded by at least one negation. Then we have

THEOREM 6.3. $\Sigma_{l}^{l}-A C_{0}+\left(\Sigma_{l}^{l}\right.$ induction $)$ is conservative over

$$
\Sigma_{l}^{l}-A C_{0}^{\#}+\left(\Sigma_{l}^{l} \text { induction }^{\#}\right)+\left(M P_{\text {arith }}\right)
$$

for formulae in $\mathscr{C}_{\text {res }}$, and similarly for $\Sigma_{l}^{l}-A C$ and $\Sigma_{l}^{l}-A C^{\#}+\left(M P_{\text {arith }}\right)$. 
To interpret the intermediate theories, let us take our forcing conditions to be finite sets

$$
P=\left\{\forall X \varphi_{1}(X), \ldots, \forall X \varphi_{k}(X)\right\}
$$

of $\Pi_{1}^{1}$ sentences with first- and second-order parameters. In order to code these parameters, conditions must be represented by second-order objects. Let $\operatorname{Tr}_{\text {arith }}^{m}$ be a truth predicate for a sufficiently large subset of the arithmetic sentences, and use this to define

$$
\operatorname{Tr}(P, U) \equiv \bigwedge_{i=1}^{k} \forall X \in U \varphi_{i}(X) .
$$

For any formula $\psi$, define

$$
P \vdash \psi \equiv \exists U(\operatorname{Tr}(P, U) \rightarrow \psi) .
$$

Then define

$$
p \Vdash \theta \equiv p \vdash \theta
$$

for atomic formulae $\theta$, and extend the forcing definition to arbitrary formulae in the usual way. Since most of the proofs from Section 5 now carry over, mutatis mutandis, I will only sketch the details below.

Lemma 6.4. Let $\varphi$ be any negative arithmetic formula. Then in $\Sigma_{l}^{I}$-A $C_{0}^{i}$ one can prove

$$
P \Vdash \varphi \leftrightarrow P \vdash \varphi .
$$

LEMMA 6.5. If $\varphi$ is any negative arithmetic formula, then the following are provable in $\Sigma_{l}^{l}-A C_{0}^{i}$ :

1. $\Vdash \exists X \varphi$ iff $\exists X \varphi$.

2. $\forall X \varphi \Vdash \forall X \varphi$.

3. If $p \Vdash \neg \forall X \varphi$ then $p \Vdash \exists W \neg \forall X \in W \varphi$.

LEMMA 6.6. In $\Sigma_{I}^{l}-A C_{0}^{i}+\left(\Sigma_{l}^{l}\right.$ induction $)$ one can prove that each axiom of $\Sigma_{I}^{l}-A C_{0}^{\#}+$ $\left(\Sigma_{l}^{l}\right.$ induction $\left.^{\#}\right)+\left(M P_{\text {arith }}\right)$ is forced; and similarly for $\Sigma_{I}^{I}-A C^{i}$ and $\Sigma_{I}^{l}-A C^{\#}+\left(M P_{\text {arith }}\right)$.

Proof. Lemma 6.5.1 takes care of the quantifier-free axioms and $\left(A C A^{\#}\right)$, and we can verify that induction is forced just as we did for first-order arithmetic, in the proof of Lemma 3.5. Lemma 6.5.3 shows that $\left(M P_{\text {arith }}\right)$ is forced as well.

Regarding $\left(\Sigma_{I}^{l}-A C^{\#}\right)$, suppose

$$
P \Vdash \forall x \exists Y \varphi(x, Y),
$$

where $\varphi$ is negative and arithmetic. This implies

$$
\forall x \exists Y P \vdash \varphi(x, Y) .
$$

Since $P \vdash \varphi(x, Y)$ is $\Sigma_{1}^{1}$, the desired conclusion follows from an application of $\left(\Sigma_{l}^{l}-A C\right)$.

If $\mathscr{D}_{\text {arith }}$ is the smallest set containing the weak $\Sigma_{1}^{1}$ formulae and closed under conjunction, disjunction, and universal and existential quantification, we have

THEOREM 6.7. $\Sigma_{l}^{l}-A C_{0}^{\#}+\left(\Sigma_{l}^{l}\right.$ induction $\left.^{\#}\right)+\left(M P_{\text {arith }}\right)$ is conservative over

$$
\Sigma_{l}^{l}-A C_{0}^{i}+\left(\Sigma_{l}^{l} \text { induction }\right)
$$

for formulae in $\mathscr{D}_{\text {arith }}$; and similarly for $\Sigma_{I}^{l}-A C^{\#}+\left(M P_{\text {arith }}\right)$ and $\Sigma_{l}^{l}-A C^{i}$.

Combining Theorems 6.3 and 6.7 we have 
THEOREM 6.8. If $\Sigma_{l}^{l}-A C_{0}+\left(\Sigma_{l}^{l}\right.$ induction) proves $\forall X \exists Y \varphi(X, Y)$, where $\varphi$ is arithmetic, then $\Sigma_{l}^{l}-A C_{0}^{i}+\left(\Sigma_{l}^{l}\right.$ induction) proves $\exists W \forall X \exists Y \in W \varphi^{N}(X, Y)$. The corresponding assertion also holds for $\Sigma_{1}^{l}-A C$ and $\Sigma_{1}^{l}-A C^{i}$.

Both classical theories are stable under the Friedman-Dragalin translation (see [14, Section 3]). As a result, here too we can recapture the theorems that are arithmetic $\Pi_{2}$.

§7. Questions. Given a proof of $\neg \neg \exists x A(x)$ in Heyting arithmetic, where $A$ is primitive recursive, one can use either the Friedman-Dragalin translation or the interpretation described above to extract a proof of $\exists x A(x)$. What can one can say about the relationship between the two methods?

In a sense, Buchholz' interpretations in [7] are more general than the ones described here, since they allow one to interpret iterations of the basic theory. An "iterated" version of $\Sigma_{I}^{l}-A C$ yields the theory $A T R_{0}$, whose main axiom is equivalent to the assertion that every set $X$ is contained in a coded model of $\Sigma_{I}^{l}-A C$ (see [4] or [21]); and the theories $K P l$ and $K P i$ can be seen as "iterated" versions of $K P \omega$, since they axiomatize segments of the constructible set hierarchy that correspond to limits (resp. admissible limits) of admissible ordinals. Can the methods described here be used to provide direct interpretations of $A T R_{0}, K P l$, and $K P i$, in intuitionistic versions thereof? Such an interpretation of $K P i$ would be particularly interesting, because at present the only means of reducing it to its intuitionistic counterpart involves an ordinal analysis.

\section{REFERENCES}

[1] Peter Aczel, The strength of Martin-Löf's intuitionistic type theory with one universe, Proceedings of the symposiums of mathematical logic, Oulu 1974 and Helsinki 1975 (Seppo Miettinen and Jouko Väänänen, editors), Department of Philosophy, University of Helsinki, 1977.

[2] - The type theoretic interpretation of constructive set theory, Logic colloquium '77 (A. Macintyre, L. Pacholski, and J. Paris, editors), North-Holland, Amsterdam, 1978, pp. 55-66.

[3] - The type theoretic interpretation of constructive set theory: choice principles, L. E. J. Brouwer centenary symposium (A. S. Troelstra and D. van Dalen, editors), North-Holland, Amsterdam, 1982, pp. 1-40.

$\rightarrow$ JEREMY AVIGAD and Richard SOMMER, The model-theoretic ordinal analysis of predicative theories, this JOURNAL, vol. 64 (1999), pp. 327-349.

[5] Jon BARWISE, Admissible sets and structures, Springer, Berlin, 1975.

[6] MiCHAEL J. BEESON, Foundations of constructive mathematics, Springer, Berlin, 1985.

[7] Wilfried BuchHOLZ, The $\Omega_{\mu+1}$-rule, [8], 1981, pp. 188-233.

[8] Wilfried Buchholz, Solomon Feferman, Wolfram Pohlers, and Wilfried Sieg, Iterated inductive definitions and subsystems of analysis: Recent proof-theoretical studies, Lecture Notes in Mathematics, no. 897, Springer, Berlin, 1981.

[9] Wolfgang Burr, Fragments of Heyting-arithmetic, preprint, 1998.

[10] Stephen A. CoOK and AlasDaIR URQuHART, Functional interpretations of feasibly constructive arithmetic, Annals of Pure and Applied Logic, vol. 63 (1993), pp. 103-200.

[11] ThIERry CoQuand and Martin Hofmann, A new method for establishing conservativity of classical systems over their intuitionistic version, Mathematical Structures in Computer Science, vol. 9 (1999), pp. 323-333.

[12] Harvey M. Friedman, Subsystems of set theory and analysis, Ph.D. thesis, Massachusetts Institute of Technology, 1967.

$[13]-\rightarrow-$, The consistency of classical set theory relative to a set theory with intuitionistic logic, this JoURNAL, vol. 38 (1973), no. 2, pp. 315-319. 
[14] - Classically and intuitionistically provable functions, Higher set theory (H. Müller and D. Scott, editors), Lecture Notes in Mathematics, no. 669, Springer, Berlin, 1978, pp. 21-27.

[15] E. GRIFFOR and M. RathJEN, The strength of some Martin-Löf type theories, Archive for Mathematical Logic, vol. 33 (1994), pp. 347-385.

[16] GERHARD JäGER, Theories for admissible sets: $A$ unifying approach to proof theory, Bibliopolis, Napoli, 1986.

[17] Per Martin-LöF, An intuitionistic theory of types, 1972, manuscript, reprinted in Twenty-five years of constructive type theory, Oxford University Press, 1998, pp. 127-172.

[18] ERIK PALMGREN, Type-theoretic interpretation of iterated, strictly positive inductive definitions, Archive for Mathematical Logic, vol. 32 (1992), pp. 75-99.

$\lfloor\rightarrow$ RoHit PARIKH, Existence and feasibility in arithmetic, this JoURNAL, vol. 36 (1971), pp. 494-508.

$\longrightarrow$ MICHAEL RATHJEN, The proof-theoretic characterization of the primitive recursive set functions, this JOURNAL, vol. 57 (1992), pp. 954-969.

[21] STEPHEN G. SIMPSON, Subsystems of second-order arithmetic, Springer, Berlin, 1998.

[22] A. S. Troelstra, Metamathematical investigation of intuitionistic arithmetic and analysis, Lecture Notes in Mathematics, no. 344, Springer, Berlin, 1973.

[23] Dirk van Dalen, Logic and structure, third ed., Springer, Berlin, 1997.

\section{DEPARTMENT OF PHILOSOPHY \\ CARNEGIE-MELLON UNIVERSITY \\ PITTSBURGH, PA 15213, USA}

E-mail: avigad@cmu.edu 